Characterising the shape and material properties of hidden targets from magnetic induction data

Ledger, Paul D and Lionheart, William RB 2013

Manchester Institute for Mathematical Sciences

School of Mathematics

The University of Manchester

\footnotetext{
Reports available from: http://eprints.maths.manchester.ac.uk/

And by contacting: The MIMS Secretary

School of Mathematics

The University of Manchester

Manchester, M13 9PL, UK
} 


\title{
Characterising the shape and material properties of hidden targets from magnetic induction data
}

\author{
P.D. Ledger ${ }^{1}$ and W.R.B Lionheart ${ }^{2}$ \\ ${ }^{1}$ College of Engineering, Swansea University, SA2 8PP UK \\ ${ }^{2}$ School of Mathematics, The University of Manchester, M13 9PL UK
}

\begin{abstract}
The purpose of this paper is to clear up a major mystery in metal detection and confirm that the engineering prediction of $\boldsymbol{H}^{T} \cdot\left(\mathcal{M}^{i n d} \boldsymbol{H}^{M}\right)$, for the sensitivity of measurements of the perturbed magnetic field to the presence of a general conducting object placed in a low frequency background field, is correct. Explicitly, $H^{T}$ is the background field generated by the transmitter coil, $\boldsymbol{H}^{M}$ is the background field generated by the receiving coil as if it were used as a transmitter and $\mathcal{M}^{\text {ind }}$ is a rank 2 polarisation tensor, which describes the shape and material properties of the object. To show this, we apply a recently derived asymptotic formula for the perturbed magnetic field due to the presence of a conducting object, which is expressed in terms of a new class of rank 4 polarisation tensors (H. Ammari, J. Chen, Z. Chen, J. Garnier and D. Volkov Target detection and characterization from electromagnetic induction data, Journal de Mathématiques Pures et Appliquées (2013) http://dx.doi.org/10.1016/j.matpur.2013.05.002). At first sight this appears to contradict the engineering prediction, however, contrary to this, we show that at most 9 rather than 81 coefficients are required to describe the rank 4 tensor for a conducting object and a further 9 are required if the object is magnetic. We then show that the rank 4 tensor does in fact reduce to a rank 2 tensor, thus providing a solid theoretical foundation for the engineering prediction. Furthermore, by combining the reduced conductivity and permeability tensors, we obtain a symmetric rank 2 tensor, which describes a general conducting object in terms of just 6 complex independent coefficients. For objects with rotational and mirror symmetries we show that the number of coefficients is still smaller. We include numerical examples to demonstrate that the new polarisation tensors can be accurately computed by solving a vector valued transmission problem by $h p-$ finite elements and include evidence to confirm that the asymptotic formula describing the perturbed fields agrees with the numerically predictions.
\end{abstract}

Index Terms-Metal detectors, Land mine detection, Polarisation tensors, Eddy currents, $h p$-Finite elements

\section{INTRODUCTION}

$\mathbf{T}$ HERE is considerable interest in being able to locate and characterise conducting objects from measurements of mutual impedance between a transmitting and a receiving coil, where the coupling is inductive rather than due to the propagation of radio waves. The most obvious examples are in metal detection, where the goal is to identify and locate a highly conducting object in a low conducting background and applications include security screening, archaeological searches, maintaining food safety as well as for land mine clearance and the detection of unexploded ordnance (UXO). There is also considerable interest in being able to produce conductivity images from multiple magnetic induction measurements, most notably in magnetic induction tomography for medical applications [1], [2] and industrial applications [3], [4]. Furthermore, eddy current sensing techniques are also commonly used for the monitoring and defect detection in steel structures such as oil pipe lines and containment vessels as well as monitoring corrosion of steel reinforcement bars in concrete structures such as bridges and buildings [5].

The detection of land mines presents a huge challenge, the United Nations (UN) estimates that "there are more than 110 million active mines [...] scattered in 68 countries with an equal number stockpiled around the world waiting to be planted" and that "every month over 2,000 people are killed or maimed by mine explosions. Most of the casualties are civilians who are killed or injured after hostilities

Manuscript received 11th October 2013; revised **. Corresponding author: P.D. Ledger (email: p.d.ledger@swansea.ac.uk). have ended" [6]. Mine clearance is a slow procedure and is extremely dangerous, the UN states that "currently accidents occur at a rate of one every 1-2,000 mines destroyed" [6]. Although metal detectors offer a portable means of detection current techniques are often not able to distinguish between benign and dangerous targets and, therefore, there is great interest in technological advancements that might increase the speed at which mines could be detected and keep accidents to a minimum.

By considering the time harmonic regime, and denoting the electric field intensity vector by $\boldsymbol{E}$ and the corresponding magnetic field intensity vector by $\boldsymbol{H}$, the changes in conductivity in a isotropic background conducting medium have been shown by Sommersalo, Isaacson and Cheney to be associated with an $\boldsymbol{E}^{a} \cdot \boldsymbol{E}^{b}$ sensitivity and the corresponding permeability changes associated with an $\boldsymbol{H}^{a} \cdot \boldsymbol{H}^{b}$ sensitivity [7], where $a$ and $b$ refer to two appropriate background solutions and $\boldsymbol{E}^{a} \cdot \boldsymbol{E}^{b}=\sum_{i=1}^{3} \boldsymbol{E}_{i}^{a} \boldsymbol{E}_{i}^{b}$ denotes the non-hermitian form of the dot product. For a free space background, Ammari, Vogelius and Volkov [8] have obtained an asymptotic formula for the perturbed field due to the presence of a small object, which can be expressed in the form of a $\boldsymbol{E}^{a} \cdot\left(\mathcal{T}\left(\epsilon_{r}^{c}\right) \boldsymbol{E}^{b}\right)$ sensitivity for an object with relative complex permittivity perturbation $\epsilon_{r}^{c}-1$ and an $\boldsymbol{H}^{a} \cdot\left(\mathcal{T}\left(\mu_{r}\right) \boldsymbol{H}^{b}\right)$ sensitivity for an object with relative permeability perturbation $\mu_{r}-1$, where $\mathcal{T}(c)$ is a general rank 2 polarisation tensor parameterised by a contrast $c$, which describes the shape and material properties of the inclusion and simplifies to a multiple of the identity tensor for a sphere. As we will shortly explain, although the perturbation in complex permittivity does include the possibility of describing 
a conducting object in a non-conducting background this result is only applicable to wave propagation (electromagnetic scattering) problems. This is in contrast to experiences for the metal detection problem where, for a single transmittermeasurement arrangement, a sensitivity of the form

$$
V^{i n d} \approx \boldsymbol{H}^{M} \cdot \boldsymbol{m}=\boldsymbol{H}^{M} \cdot\left(\mathcal{M}^{i n d} \boldsymbol{H}^{T}\right),
$$

has been reported to be associated with conductivity perturbations in a low (often assumed to be non-conducting) background [9]-[12]. In the above, $\boldsymbol{H}^{T}\left(\equiv \boldsymbol{H}^{a}\right)$ is the background magnetic field generated by the transmitter coil evaluated at the position of the target and $\boldsymbol{H}^{M}\left(\equiv \boldsymbol{H}^{b}\right)$ is the background field generated by the receiving coil, as if it were used as a transmitter, evaluated at the position of the target. Furthermore, $\boldsymbol{m}$ is the induced magnetic dipole moment and it has been suggested that $\mathcal{M}^{\text {ind }}$ is some complex (symmetric) rank 2 polarisation tensor, which has been predicted to describe the shape and the material properties of the object in a similar way to $\mathcal{T}(c)$. Engineers have also felt comfortable with (1) as it appears to reflect the well known Lorentz reciprocity principal, which approximately states that the response of a system is unchanged when the transmitter and measurer are interchanged [13]. Furthermore, in the classical problem of describing the eddy currents in a conducting (magnetic) sphere placed in a uniform magnetic field (eg [14], [15]), this allows us to describe the perturbed field outside the object, and in this case, we can identity that the polarisation tensor for a conducting (magnetic) sphere is a scalar multiple of the identity tensor. An analytical solution also exists for a sphere in the time-varying field that is generated by a coil [16], and in this case we can also interpret the perturbed field outside the object in terms of an induced magnetic dipole moment and, notably, the same polarisation tensor. Experiments have been carried out to measure the response from cylindrical, spherical and ellipsoidal bodies [9], [10] and have examined how this response is affected by the size, frequency and the object's material properties. But, apart from the case of a sphere, it was not known whether such a relationship does in fact hold in general.

The previous justification for (1), and the existence of such a rank 2 polarisation tensor $\mathcal{M}^{\text {ind }}$, appears to stem from the related problem of characterising the perturbation of far field electric and magnetic fields, caused by the presence of a (small) simply connected dielectric or magnetic object (at low-frequencies). Early results by Kleinman [17], [18] related the leading order term for the perturbed (scattered fields) to equivalent dipole moments for the case when $k \rightarrow 0$ and $r \rightarrow \infty$, where $r$ is the distance from the object to the point of observation, $k=\omega \sqrt{\epsilon_{0} \mu_{0}}$ is the free space wave number, $\omega$ is the angular frequency and $\mu_{0}, \epsilon_{0}$ are the free space permeability and permittivity, respectively. Later it was shown how the moments could be expressed in terms of the dielectric and magnetic polarisation tensors multiplied by the incident electric and magnetic fields, respectively, evaluated at the position of the centre of the object. In this case, the dielectric and magnetic polarisation tensors both take the form of a general rank 2 symmetric polarisation tensor $\mathcal{T}(c)$ that is parameterised by the contrast in relative permittivity $c=\epsilon_{r}:=\epsilon_{*} / \epsilon_{0}$ and relative permeability $c=\mu_{r}:=\mu_{*} / \mu_{0}$, respectively, where $*$ indicates the values of the object [19][21]. It is known that the general polarisation tensor, for a given contrast, can be computed numerically by solving an auxiliary transmission problem and also has a known analytical solution for spherical and ellipsoidal objects [21], [22]. By taking appropriate limiting values of $\epsilon_{r}$ and $\mu_{r}$, the far field perturbation caused by the presence of a perfectly conducting object can be described [20], [21]. Furthermore, upon taking the dot product with an appropriate moment, the results can be interpreted in terms of the aforementioned $\boldsymbol{E} \cdot\left(\mathcal{T}\left(\epsilon_{r}\right) \boldsymbol{E}^{M}\right)$ and $\boldsymbol{H}^{T} \cdot\left(\mathcal{T}\left(\mu_{r}\right) \boldsymbol{H}^{M}\right)$ sensitivities for wave propagation (electromagnetic scattering) rather than magnetic induction.

The leading order terms that describe near and far field perturbations in electromagnetic fields as $k \rightarrow 0$ have been investigated by Baum [11], but these results omit the object size and are expressed in terms of dipole moments rather than polarisation tensors. In [23], motivated by results in electromagnetic scattering, Baum instead expresses the results in terms of rank 2 polarisation tensors multiplied by the incident field at the centre of the object, but does not give an explicit formula for their computation. He suggests that the polarisation tensor associated with a conducting object is frequency dependent and illustrates this by the known result for a spherical object. In [20], [21] it is suggested that, at low-frequencies, the perturbed far magnetic field caused by the presence of conducting object can be expressed in terms of a dielectric tensor $\mathcal{T}\left(\epsilon_{r}^{c}\right)$ and a magnetic polarisation $\mathcal{T}\left(\mu_{r}\right)$, where $\epsilon_{r}^{c}=1 / \epsilon_{0}\left(\epsilon_{*}-\mathrm{i} \sigma_{*} / \omega\right)$ and $\sigma_{*}$ is the object's conductivity. Given that these are supposed to be applicable for the low frequency limit, one might expect these results to be applicable to magnetic induction, but, using this parameterisation, would indicate that the far field perturbation in the magnetic field, due to the presence of a conducting object, would be associated with an electric dipole moment, rather than the expected magnetic dipole moment, and hence a $\boldsymbol{E}^{T} \cdot\left(\mathcal{T}\left(\epsilon_{r}^{c}\right) \boldsymbol{E}^{M}\right)$ sensitivity rather than a $\boldsymbol{H}^{T} \cdot\left(\mathcal{M}^{\text {ind }} \boldsymbol{H}^{M}\right)$ sensitivity.

The result of Ammari, Vogelius and Volkov [8] includes the object's size $\alpha$ and the leading order terms they obtain rigorously describes the near field perturbation of the magnetic field in a bounded domain when a small simply connected conducting object is placed in a non-conducting background. They obtain that the perturbed magnetic field can be expressed in terms of $\mathcal{T}\left(\epsilon_{r}^{c}\right)$ and $\mathcal{T}\left(\mu_{r}\right)$ multiplied by the incident electric and magnetic fields, respectively, evaluated at the position of the centre of the object. However, their result is applicable to the propagation of waves in electromagnetic scattering rather than magnetic induction as they consider the case where $\alpha \rightarrow$ 0 , for fixed $k$. Moreover, their result can be expressed in the form of a $\boldsymbol{E}^{T} \cdot\left(\mathcal{T}\left(\epsilon_{r}^{c}\right) \boldsymbol{E}^{M}\right)$ type sensitivity associated with $\epsilon_{r}^{c}$ different from unity.

The result of Ammari and Volkov [24] rigorously shows the correct far field behaviour of the perturbed far field electric field in terms of $\mathcal{T}\left(\epsilon_{r}\right)$ and $\mathcal{T}\left(\mu_{r}\right)$ multiplied by the incident electric and magnetic fields, respectively, evaluated at the centre of the object as $\alpha \rightarrow 0$ and $r \rightarrow \infty$ for a simply 
connected object and the electromagnetic scattering problem. We have also contributed to this topic by providing two rigorous asymptotic formulae whose leading order terms describe the perturbations of the electric and magnetic fields [25] in terms of $\mathcal{T}\left(\epsilon_{r}\right)$ and $\mathcal{T}\left(\mu_{r}\right)$ for dielectric or magnetic objects as $\max (\alpha / r, k \alpha) \rightarrow 0$ and the second in terms of $\mathcal{T}\left(\epsilon_{r}^{c}\right)$ and $\mathcal{T}\left(\mu_{r}\right)$ for dielectric, magnetic or conducting objects as $\max (\alpha / r, \alpha) \rightarrow 0$. The former contains terms that dominate at different distances and, by taking the limit as $k \rightarrow 0$, for fixed $r$, the electrostatic and magnetostatic behaviour for a small object can be recovered, on the other hand, fixing $k$ and taking the limit as $r \rightarrow \infty$ recovers the electromagnetic scattering behaviour of Ammari and Volkov [24]. The latter is also applicable to the electromagnetic scattering (wave propagation) with fixed $k$, is consistent with the results of Ammari, Vogelius and Volkov [8] showing an $\boldsymbol{E}^{T} \cdot\left(\mathcal{T}\left(\epsilon_{r}^{c}\right) \boldsymbol{E}^{M}\right)$ sensitivity associated with conductivity perturbations, and, consequently, does not explain magnetic induction. We also discuss in detail why the perturbed fields, in the case of a conducting object for low-frequencies, can not be expressed in terms of the general polarisation tensor parameterised by $\epsilon_{r}^{c}$, which was previously advocated by [20], [21].

Ammari, Chen, Chen, Garnier and Volkov [26] have recently obtained an asymptotic expansion, which, for the first time, correctly describes the perturbed magnetic field as $\alpha \rightarrow 0$ for a conducting (possibly magnetic and multiply connected) object in the presence of a low-frequency background magnetic field, generated by a coil with an alternating current. Rather than consider the limit as $k \rightarrow 0$ for the full timeharmonic Maxwell system they instead consider the eddy current model, where the displacement currents are neglected. The leading order term they obtain is written in terms of two new polarisation tensors, called the permeability and conductivity tensors, which have different ranks, and the background magnetic field evaluated at the position of the centre of the object. An algorithm for identifying conducting objects from induction data based on a process of classification by matching against a library of precomputed polarisation tensors has also recently been proposed [27].

In this contribution, we explain how the results of Ammari et al. [26] can be applied to understanding the mystery of metal detection. We show that the sensitivity of the perturbed magnetic field measurements to conductivity or permeability changes does in fact reduce to the form $\boldsymbol{H}^{T} \cdot\left(\mathcal{M}^{\text {ind }} \boldsymbol{H}^{M}\right)$ that the engineering community is used to, where $\mathcal{M}^{\text {ind }}$ is a rank 2 symmetric tensor. Moreover, when the object is spherical the tensor is a scalar multiple of the identity tensor. Our new contributions include considering, in detail, the properties of the conductivity and permeability tensors introduced by Ammari et al.. These studies enable us to show that, in practice, each of the tensors can be represented by just 9 independent coefficients. We show that it is also possible to express the perturbed field in terms of a reduced rank 2 conductivity tensor and introduce a symmetric rank 2 tensor, which defines a general object in terms of just 6 independent coefficients. For a simple object, with rotational or mirror symmetries, we show that the number of independent coefficients is still fewer. These results have important consequences for characterising conducting objects of different shapes. We include a description of an efficient numerical approach for accurately computing these new tensors, which is based on the regularised eddy current formulation using $h p$-finite elements presented in [28]. We also present simulations, which indicate an excellent agreement between the asymptotic formula of Ammari et al. using the numerically computed polarisation tensors and the fields obtained from solving the full eddy current problem, for a range of case studies.

The presentation of the paper proceeds as follows: In Section II we describe the mathematical model and simplifying assumptions made about the problem under consideration. Then, in Section III, we apply the asymptotic formula of Ammari et al. [26] to the metal detection problem and show an alternative form where their their rank 4 conductivity and rank 2 permeability tensors can be expressed as a single rank 4 tensor. In Section IV, we discuss the properties of the tensors, which allow them to be represented by a smaller number of independent coefficients and show that the perturbed magnetic field can, alternatively, be expressed in terms of a single symmetric rank 2 tensor. In Section V, we discuss how further reductions in the number of independent coefficients can be obtained if the object is rotationally symmetric or has mirror symmetries and the additional simplifications for a sphere. Then, in Section VI, we interpret how reciprocity can be understood for the asymptotic formula of Ammari et al. and in Section VII we describe how the independent coefficients of the tensors can be recovered from practical measurement data. In Section VIII, we describe an approach to the numerical computation of the polarisation tensors based on $h p$-finite elements, in Section IX, we present a series of numerical results to validate this approach and finish with our concluding remarks in $\mathrm{X}$.

\section{Mathematical Model}

The relevant mathematical model is that of the eddy current problem where the geometry, frequency and material parameters are such that the displacement currents in the Maxwell system can be neglected. This is often justified on the basis that $\sqrt{\epsilon_{*} \mu_{*}} \alpha \omega \ll 1$ or $\epsilon_{*} \omega / \sigma_{*} \ll 1$ where $\omega$ denotes the angular frequency, $\alpha$ is a suitable length scale and $\epsilon_{*}, \mu_{*}$ and $\sigma_{*}$ denote the permittivity, permeability and conductivity of the object, respectively. A more rigorous justification of the eddy current model appears in [29]. In [30] the effect of the shape of the conductor on the validity of the eddy current model is discussed. The depth of penetration of the magnetic field in a conducting object is described by its skin depth, $s:=\sqrt{\frac{2}{\omega \mu_{0} \sigma_{*}}}$, and, introducing a parameter $\nu:=2 \alpha^{2} / s^{2}$, it is possible to describe the mathematical model of interest, which relates to when $\nu=O(1)$ and $\mu_{*} / \mu_{0}=O(1)$ as $\alpha \rightarrow 0$ [26]. This model is applicable to homogeneous isotropic materials, which have a linear constitutive relationship between the magnetic flux density and magnetic field and have a skin depth of the same order as the object size. It then follows that $\sigma_{*}$ and $\mu_{*}$ are then constants that describe non-ferrous metallic conductors and appropriate magnetic materials.

Following Ammari et al. [26] we consider an object of the form $B_{\alpha}=\boldsymbol{z}+\alpha B$, which means that the physical object can 
be expressed in terms of unit object $B$ placed at the origin, scaled by the object size $\alpha$ and translated by the vector $\boldsymbol{z}$, and introduce

$$
\mu_{\alpha}=\left\{\begin{array}{ll}
\mu_{*} & \text { in } B_{\alpha} \\
\mu_{0} & \text { in } \mathbb{R} \backslash B_{\alpha}
\end{array} \quad \sigma_{\alpha}=\left\{\begin{array}{ll}
\sigma_{*} & \text { in } B_{\alpha} \\
0 & \text { in } \mathbb{R} \backslash B_{\alpha}
\end{array},\right.\right.
$$

where the subscript on $\mu_{\alpha}$ and $\sigma_{\alpha}$ is removed when considering the unit object case. We remark that the background medium is assumed to be non-conducting, which is a reasonable approximation to make for buried objects provided that the contrast between the object and the surrounding soil is sufficiently high. The time harmonic fields $\boldsymbol{E}_{\alpha}$ and $\boldsymbol{H}_{\alpha}$ that result from a time varying current source, with volume current density $\boldsymbol{J}_{0}$ and $\nabla \cdot \boldsymbol{J}_{0}=0$ in $\mathbb{R}^{3}$, and their interaction with the object $B_{\alpha}$, satisfy the eddy current equations

$$
\begin{array}{cl}
\nabla \times \boldsymbol{E}_{\alpha}=\mathrm{i} \omega \mu_{\alpha} \boldsymbol{H}_{\alpha} & \text { in } \mathbb{R}^{3}, \\
\nabla \times \boldsymbol{H}_{\alpha}=\sigma_{\alpha} \boldsymbol{E}_{\alpha}+\boldsymbol{J}_{0} & \text { in } \mathbb{R}^{3}, \\
\boldsymbol{E}_{\alpha}(\boldsymbol{x})=O\left(|\boldsymbol{x}|^{-1}\right), \boldsymbol{H}_{\alpha}(\boldsymbol{x})=O\left(|\boldsymbol{x}|^{-1}\right) & \text { as }|\boldsymbol{x}| \rightarrow \infty .
\end{array}
$$

On the other hand, the fields $\boldsymbol{E}_{0}$ and $\boldsymbol{H}_{0}$ that result from time varying current source, in the absence of an object, satisfy

$$
\begin{array}{cl}
\nabla \times \boldsymbol{E}_{0}=\mathrm{i} \omega \mu_{0} \boldsymbol{H}_{0} & \text { in } \mathbb{R}^{3}, \\
\nabla \times \boldsymbol{H}_{0}=\boldsymbol{J}_{0} & \text { in } \mathbb{R}^{3}, \\
\boldsymbol{E}_{0}(\boldsymbol{x})=O\left(|\boldsymbol{x}|^{-1}\right), \boldsymbol{H}_{0}(\boldsymbol{x})=O\left(|\boldsymbol{x}|^{-1}\right) & \text { as }|\boldsymbol{x}| \rightarrow \infty .
\end{array}
$$

The task is to describe the perturbation $\boldsymbol{H}_{\alpha}(\boldsymbol{x})-\boldsymbol{H}_{0}(\boldsymbol{x})$, caused by the presence of the object $B_{\alpha}$, in terms of a polarisation tensor. This is made challenging due to the range of important parameters controlling the problem namely, the skin depth, $s$, the frequency, $\omega$, the object size, $\alpha$, and the point of observation $\boldsymbol{r}:=\boldsymbol{x}-\boldsymbol{z}$, relative to the position of the object.

\section{THE PERTURBED MAGNETIC FIELD EXPRESSED IN TERMS OF A POLARISATION TENSOR}

For the mathematical model described above, Ammari et al. [26] have derived an asymptotic formula that describes the perturbed magnetic field at positions $\boldsymbol{x}$ away from $\boldsymbol{z}$, due to the presence of object $B_{\alpha}$, as $\alpha \rightarrow 0:^{1}$

$$
\begin{aligned}
& \left(\boldsymbol{H}_{\alpha}-\boldsymbol{H}_{0}\right)(\boldsymbol{x})= \\
& -\frac{\mathrm{i} \nu \alpha^{3}}{2} \sum_{i=1}^{3} \boldsymbol{H}_{0}(\boldsymbol{z})_{i} \int_{B} \boldsymbol{D}_{\boldsymbol{x}}^{2} G(\boldsymbol{x}, \boldsymbol{z}) \boldsymbol{\xi} \times\left(\boldsymbol{\theta}_{i}+\hat{\boldsymbol{e}}_{i} \times \boldsymbol{\xi}\right) \mathrm{d} \boldsymbol{\xi}+ \\
& \alpha^{3}\left(1-\frac{\mu_{0}}{\mu_{*}}\right) \sum_{i=1}^{3} \boldsymbol{H}_{0}(\boldsymbol{z})_{i} \\
& \boldsymbol{D}_{\boldsymbol{x}}^{2} G(\boldsymbol{x}, \boldsymbol{z}) \int_{B}\left(\hat{\boldsymbol{e}}_{i}+\frac{1}{2} \nabla \times \boldsymbol{\theta}_{i}\right) \mathrm{d} \boldsymbol{\xi}+\boldsymbol{R}(\boldsymbol{x}),
\end{aligned}
$$

where $G(\boldsymbol{x}, \boldsymbol{z})=\frac{1}{4 \pi|\boldsymbol{x}-\boldsymbol{z}|}$ is the free space Laplace Green's function and $|\boldsymbol{R}(\boldsymbol{x})| \leqslant C \alpha^{4}\left\|\boldsymbol{H}_{0}\right\|_{W^{2, \infty}\left(B_{\alpha}\right)}$ such that $\boldsymbol{R}(\boldsymbol{x})=$ $O\left(\alpha^{4}\right)$ is a small remainder term. Furthermore, $\hat{\boldsymbol{e}}_{i}$ is a unit

${ }^{1}$ Note that $I_{3}$ on page 10 of [26] requires that $\left.\nabla \boldsymbol{x} G(\boldsymbol{x}, \alpha \boldsymbol{\xi}+\boldsymbol{z})\right)-$ $\nabla \boldsymbol{x} G(\boldsymbol{x}, \boldsymbol{z})-\alpha \boldsymbol{D}_{\boldsymbol{x}}^{2} G(\boldsymbol{x}, \boldsymbol{z}) \boldsymbol{\xi}=O\left(\alpha^{2}\right)$, but instead the correct choice is $\left.\nabla_{\boldsymbol{x}} G(\boldsymbol{x}, \alpha \boldsymbol{\xi}+\boldsymbol{z})\right)-\nabla \boldsymbol{x} G(\boldsymbol{x}, \boldsymbol{z})+\alpha \boldsymbol{D}_{\boldsymbol{x}}^{2} G(\boldsymbol{x}, \boldsymbol{z}) \boldsymbol{\xi}=O\left(\alpha^{2}\right)$, which adds a minus sign to the first term of (9). vector for the $i$ th Cartesian coordinate direction, $\boldsymbol{H}_{0}(\boldsymbol{z})_{i}$ denotes the $i$ th element of $\boldsymbol{H}_{0}(\boldsymbol{z})$ and $\boldsymbol{\theta}_{i}, i=1,2,3$, is the solution to the transmission problem

$$
\begin{array}{rlrl}
\nabla_{\boldsymbol{\xi}} \times \mu^{-1} \nabla_{\boldsymbol{\xi}} \times \boldsymbol{\theta}_{i}- & & \\
\mathrm{i} \omega \sigma \alpha^{2} \boldsymbol{\theta}_{i} & =\mathrm{i} \omega \sigma \alpha^{2} \hat{\boldsymbol{e}}_{i} \times \boldsymbol{\xi} & & \text { in } B \cup B^{c}, \\
\nabla_{\boldsymbol{\xi}} \cdot \boldsymbol{\theta}_{i} & =0 & & \text { in } B^{c}, \\
{\left[\boldsymbol{\theta}_{i} \times \hat{\boldsymbol{n}}\right]_{\Gamma}} & =\mathbf{0} & & \text { on } \Gamma, \\
{\left[\mu^{-1} \nabla_{\boldsymbol{\xi}} \times \boldsymbol{\theta}_{i} \times \hat{\boldsymbol{n}}\right]_{\Gamma}} & = & & \\
-2\left[\mu^{-1}\right]_{\Gamma} \hat{\boldsymbol{e}}_{i} \times \hat{\boldsymbol{n}} & & \text { on } \Gamma, \\
\boldsymbol{\theta}_{i}(\boldsymbol{\xi}) & =O\left(|\boldsymbol{\xi}|^{-1}\right) & & \text { as }|\boldsymbol{\xi}| \rightarrow \infty,
\end{array}
$$

where $\boldsymbol{\xi}$ is measured from the centre of $B$. An alternative form of this result is as follows

$$
\begin{gathered}
\left(\boldsymbol{H}_{\alpha}-\boldsymbol{H}_{0}\right)(\boldsymbol{x})_{j}=\boldsymbol{D}_{\boldsymbol{x}}^{2} G(\boldsymbol{x}, \boldsymbol{z})_{\ell m} \mathcal{P}_{\ell m j i} \boldsymbol{H}_{0}(\boldsymbol{z})_{i}+ \\
\boldsymbol{D}_{\boldsymbol{x}}^{2} G(\boldsymbol{x}, \boldsymbol{z})_{j \ell} \mathcal{N}_{\ell i} \boldsymbol{H}_{0}(\boldsymbol{z})_{i}+\boldsymbol{R}(\boldsymbol{x})_{j},
\end{gathered}
$$

where Einstein summation convention is used and

$$
\begin{aligned}
\mathcal{P}_{\ell m j i} & :=-\frac{\mathrm{i} \nu \alpha^{3}}{2} \hat{\boldsymbol{e}}_{j} \cdot\left(\hat{\boldsymbol{e}}_{\ell} \times \int_{B} \xi_{m}\left(\boldsymbol{\theta}_{i}+\hat{\boldsymbol{e}}_{i} \times \boldsymbol{\xi}\right) \mathrm{d} \boldsymbol{\xi}\right), \\
\mathcal{N}_{\ell i} & :=\alpha^{3}\left(1-\frac{\mu_{0}}{\mu_{*}}\right) \int_{B}\left(\hat{\boldsymbol{e}}_{\ell} \cdot \hat{\boldsymbol{e}}_{i}+\frac{1}{2} \hat{\boldsymbol{e}}_{\ell} \cdot \nabla \times \boldsymbol{\theta}_{i}\right) \mathrm{d} \boldsymbol{\xi} .
\end{aligned}
$$

The rank 4 tensor $\mathcal{P}$ and the rank 2 tensor $\mathcal{N}$ are the conductivity and permeability tensors of Ammari et al. [26], respectively, where we have chosen to chosen to make the ranks of the tensors explicit. Note that to simplify the notation, and avoid confusion when manipulating the tensors, we use $\xi_{m}$ to denote the $m$ th element of $\boldsymbol{\xi}$ rather than $\boldsymbol{\xi}_{m}$. In general, a rank 4 tensor is defined by 81 coefficients and a rank 2 tensor by 9 coefficients, but, in the next section, we will explain that, in practice, these tensors can be defined by a smaller number of independent coefficients. A further alternative representation can be obtained by extending $\mathcal{N}$ to the fourth order tensor

$$
\widehat{\widehat{\mathcal{N}}}_{k m j i}:=\delta_{k j} \delta_{m \ell} \mathcal{N}_{\ell i},
$$

where $\delta_{k j}$ is the Kronecker delta and we use a hat to indicate when the rank of a tensor is extended by 1 and a check when the rank is reduced by 1 . It then follows, by introducing the new total rank 4 tensor

$$
\mathcal{M}_{\ell m j i}:=\mathcal{P}_{\ell m j i}+\widehat{\widehat{\mathcal{N}}}_{\ell m j i},
$$

that we can express (9) in the alternative compact form

$$
\begin{aligned}
\left(\boldsymbol{H}_{\alpha}-\boldsymbol{H}_{0}\right)(\boldsymbol{x})_{j}= & \boldsymbol{D}_{\boldsymbol{x}}^{2} G(\boldsymbol{x}, \boldsymbol{z})_{\ell m} \mathcal{M}_{\ell m j i} \boldsymbol{H}_{0}(\boldsymbol{z})_{i}+ \\
& \boldsymbol{R}(\boldsymbol{x})_{j},
\end{aligned}
$$

which now only involves $\mathcal{M}$.

\section{Properties of the tensors}

In this section we explore the properties of $\mathcal{P}, \mathcal{N}$ and $\mathcal{M}$, which will be fruitful for understanding the number of independent coefficients that are required for representing these tensors and, hence, characterising objects of different types. 


\section{A. Properties of the conductivity tensor $\mathcal{P}$}

Introducing $\beta:=-\frac{\mathrm{i} \nu \alpha^{3}}{2}$, then we remark that the tensor $\mathcal{P}$ can be written in the alternative form

$$
\begin{aligned}
\mathcal{P}_{\ell m j i} & =\beta \delta_{t j} \varepsilon_{t \ell s} \int_{B} \xi_{m}\left(\theta_{s i}+\varepsilon_{s p q} \delta_{p i} \xi_{q}\right) \mathrm{d} \boldsymbol{\xi} \\
& =\beta \varepsilon_{j \ell s} \int_{B} \xi_{m}\left(\theta_{s i}+\varepsilon_{s i q} \xi_{q}\right) \mathrm{d} \boldsymbol{\xi} \\
& =\varepsilon_{j \ell s} \check{\mathcal{P}}_{m s i}=\varepsilon_{j \ell s} \mathcal{C}_{m s i},
\end{aligned}
$$

where $\theta_{s i}$ are the elements of the rank 2 tensor whose columns are $\boldsymbol{\theta}_{i}, \varepsilon_{j \ell s}$ is the alternating tensor and $\mathcal{C}$ is a rank 3 tensor, which is defined as

$$
\begin{aligned}
\mathcal{C}_{m s i}: & =\beta \int_{B} \xi_{m}\left(\theta_{s i}+\varepsilon_{s p q} \delta_{p i} \xi_{q}\right) \mathrm{d} \boldsymbol{\xi} \\
& =-\frac{\mathrm{i} k \alpha^{3}}{2} \hat{\boldsymbol{e}}_{s} \cdot \int_{B} \xi_{m}\left(\boldsymbol{\theta}_{i}+\hat{\boldsymbol{e}}_{i} \times \boldsymbol{\xi}\right) \mathrm{d} \boldsymbol{\xi},
\end{aligned}
$$

such that $\mathcal{C}_{m s i}=\frac{1}{2} \varepsilon_{s j \ell} \mathcal{P}_{\ell m j i}$. Note that in the above, and in the sequel, repeated indices in $\mathcal{C}_{m s i}$ do not imply summation. Both $\varepsilon$ and $\mathcal{C}$ are, strictly speaking, rank 3 tensor densities and their product in (14) results in a normal rank 4 tensor. Another interpretation is obtained by considering

$$
\mathcal{P}_{\ell m j i}=\varepsilon_{j \ell s} \mathcal{C}_{m s i}=-\varepsilon_{\ell j s} \mathcal{C}_{m s i}=-\mathcal{P}_{j m \ell i},
$$

which means that $\mathcal{P}_{\ell m j i}$ is skew-symmetric with respect to the indices $\ell$ and $j$. This means that, instead of the 81 coefficients normally required for a rank 4 tensor, only at most 27 independent coefficients are required for $\mathcal{P}$. Moreover, in Appendix A, we show that, for a general object, the tensor $\mathcal{C}$ is skew symmetric in the first 2 indices so that $\mathcal{C}_{m s i}=-\mathcal{C}_{s m i}$, which means that it can be represented by just 9 independent coefficients: $\mathcal{C}_{123}=-\mathcal{C}_{213}, \mathcal{C}_{132}=-\mathcal{C}_{312}, \mathcal{C}_{231}=-\mathcal{C}_{321}$, $\mathcal{C}_{121}=-\mathcal{C}_{211}, \mathcal{C}_{212}=-\mathcal{C}_{122}, \mathcal{C}_{131}=-\mathcal{C}_{311}, \mathcal{C}_{232}=-\mathcal{C}_{322}$, $\mathcal{C}_{313}=-\mathcal{C}_{133}$ and $\mathcal{C}_{323}=-\mathcal{C}_{233}$. This means that the number of independent coefficients for $\mathcal{P}$ reduces to at most 9 . The skew symmetry of $\mathcal{C}$ also means that the remaining independent coefficients can be arranged as a rank 2 tensor

$$
\begin{aligned}
\check{\mathcal{P}}_{n i} & :=\frac{1}{2} \varepsilon_{n m s} \check{\mathcal{P}}_{m s i}=\frac{1}{4} \varepsilon_{n m s} \varepsilon_{s j l} \mathcal{P}_{\ell m j i} \\
& =\frac{1}{2} \varepsilon_{n m s} \mathcal{C}_{m s i}=\frac{\beta}{2} \hat{\boldsymbol{e}}_{n} \cdot \int_{B} \boldsymbol{\xi} \times\left(\boldsymbol{\theta}_{i}+\hat{\boldsymbol{e}}_{i} \times \boldsymbol{\xi}\right) \mathrm{d} \boldsymbol{\xi} \\
& =: \check{\mathcal{C}}_{n i},
\end{aligned}
$$

such that $\mathcal{C}_{m s i}=\varepsilon_{m s k} \breve{\mathcal{C}}_{k i}$.

We now take an alternative perspective and examine how many coefficients of $\mathcal{P}$ influence the perturbed field $\left(\boldsymbol{H}_{\alpha}-\right.$ $\left.\boldsymbol{H}_{0}\right)(\boldsymbol{x})$. This can be understood by closer inspection of the contraction between $\boldsymbol{D}_{\boldsymbol{x}}^{2} G(\boldsymbol{x}, \boldsymbol{z})_{\ell m}$ and $\mathcal{P}_{\ell m j i}$ where, explicitly,

$$
\begin{aligned}
\boldsymbol{D}_{\boldsymbol{x}}^{2} G(\boldsymbol{x}, \boldsymbol{z})_{\ell m} & =\frac{1}{4 \pi r^{3}}\left(3(\hat{\boldsymbol{r}} \otimes \hat{\boldsymbol{r}})_{\ell m}-\delta_{l m}\right) \\
& =\frac{1}{4 \pi r^{3}}\left(3 \hat{r}_{\ell} \hat{r}_{m}-\delta_{l m}\right),
\end{aligned}
$$

$\boldsymbol{r}=\boldsymbol{x}-\boldsymbol{z}, r=|\boldsymbol{r}|$ and $\hat{\boldsymbol{r}}=\boldsymbol{r} / r$. Thus, provided the position of observation is away from the object (ie $\boldsymbol{x} \neq \boldsymbol{z}$ ), it is clear that $\boldsymbol{D}_{\boldsymbol{x}}^{2} G(\boldsymbol{x}, \boldsymbol{z})$ has the two important properties, namely that

$$
\begin{aligned}
\boldsymbol{D}_{\boldsymbol{x}}^{2} G(\boldsymbol{x}, \boldsymbol{z})_{\ell m} & =\boldsymbol{D}_{\boldsymbol{x}}^{2} G(\boldsymbol{x}, \boldsymbol{z})_{m \ell}, \\
\operatorname{tr}\left(\boldsymbol{D}_{\boldsymbol{x}}^{2} G(\boldsymbol{x}, \boldsymbol{z})\right) & =\boldsymbol{D}_{\boldsymbol{x}}^{2} G(\boldsymbol{x}, \boldsymbol{z})_{\ell \ell}=0 .
\end{aligned}
$$

Although we could consider the impact of the symmetry of $\boldsymbol{D}_{\boldsymbol{x}}^{2} G(\boldsymbol{x}, \boldsymbol{z})$ on $\mathcal{P}$, we instead immediately state the more important result

$$
\begin{aligned}
\boldsymbol{D}_{\boldsymbol{x}}^{2} G(\boldsymbol{x}, \boldsymbol{z})_{\ell m} \mathcal{P}_{\ell m j i} & =\boldsymbol{D}_{\boldsymbol{x}}^{2} G(\boldsymbol{x}, \boldsymbol{z})_{\ell m} \varepsilon_{j \ell s} \varepsilon_{m s k} \breve{\mathcal{C}}_{k i} \\
& =-\boldsymbol{D}_{\boldsymbol{x}}^{2} G(\boldsymbol{x}, \boldsymbol{z})_{j k} \breve{\mathcal{C}}_{k i}
\end{aligned}
$$

which follows from the representation of $\mathcal{P}$ in terms of $\breve{\mathcal{C}}$ and the trace free nature of $\boldsymbol{D}_{\boldsymbol{x}}^{2} G(\boldsymbol{x}, \boldsymbol{z})$. This means that the perturbed field for a conducting object can be described in terms of a rank 2 tensor and is only influenced by its 9 independent coefficients.

In the case of a non-magnetic object, with $\mu_{*}=\mu_{0}$, we have a further reduction in the number of independent coefficients. In Appendix B we show that, in this case, the tensor $\breve{\mathcal{C}}$ is symmetric and, therefore, $\breve{\mathcal{C}}, \mathcal{C}$ and $\mathcal{P}$ are just defined by 6 independent coefficients.

\section{B. Properties of the permeability tensor $\mathcal{N}$}

For a general object, 9 independent coefficients are required to define a general $\mathcal{N}$, and hence $\widehat{\widehat{\mathcal{N}}}$.

If the object is non conducting and magnetic so that $\sigma_{*}=0$ and $\mu_{*} \neq \mu_{0}$, then, provided that $B_{\alpha}$ is a simply connected smooth object, the tensor $\mathcal{N}$ reduces to the symmetric general polarisation tensor parameterised by a contrast $\mu_{r}:=\mu_{*} / \mu_{0}$ in the object. This, in turn, also agrees with the first order generalised polarisation tensor of Ammari and Kang [22] and the aforementioned general polarisation tensor

$$
\begin{aligned}
(\mathcal{N})_{i j} & =\mathcal{T}\left(\mu_{r}\right)_{i j} \\
& =\left(\mu_{r}-1\right)|B| \delta_{i j}+\left(\mu_{r}-1\right)^{2} \int_{\Gamma} \hat{\boldsymbol{n}} \cdot \nabla \phi_{i} \xi_{j} \mathrm{~d} \boldsymbol{\xi},
\end{aligned}
$$

where $\phi_{i}, i=1,2,3$, satisfies the transmission problem

$$
\begin{array}{rlrl}
\nabla^{2} \phi_{i} & =0 & & \text { in } B \cup B^{c}, \\
{\left[\phi_{i}\right]_{\Gamma}} & =0 & & \text { on } \Gamma, \\
\left.\frac{\partial \phi_{i}}{\partial \hat{\boldsymbol{n}}}\right|_{+}-\left.\mu_{r} \frac{\partial \phi_{i}}{\partial \hat{\boldsymbol{n}}}\right|_{-} & =\frac{\partial \xi_{i}}{\partial \hat{\boldsymbol{n}}} & & \text { on } \Gamma, \\
\phi_{i} \rightarrow 0 & & \text { as }|\boldsymbol{\xi}| \rightarrow \infty .
\end{array}
$$

As $\mathcal{N}$ is symmetric for a non-conducting object it is defined by 6 independent coefficients for this case.

\section{Properties of the total polarisation tensor $\mathcal{M}$}

The components $\mathcal{P}_{\ell m j i}$ and $\widehat{\widehat{\mathcal{N}}}_{\ell m j i}$ (as defined in (12)) of $\mathcal{M}_{\ell m j i}$ satisfy the properties as described in Sections IV-A and IV-B and form the disjoint skew-symmetric and symmetric parts of the total tensor with respect to indices $\ell$ and $j$, respectively. For a general object, we have shown that at most 
9 independent coefficients of $\mathcal{P}$ and $\widehat{\widehat{\mathcal{N}}}$, respectively, influence the perturbed field. However, by using (17), we can also write

$$
\begin{aligned}
\boldsymbol{D}_{\boldsymbol{x}}^{2} G(\boldsymbol{x}, \boldsymbol{z})_{\ell m} \mathcal{M}_{\ell m j i} & =\boldsymbol{D}_{\boldsymbol{x}}^{2} G(\boldsymbol{x}, \boldsymbol{z})_{j m}\left(-\check{\mathcal{C}}_{m i}+\mathcal{N}_{m i}\right) \\
& =\boldsymbol{D}_{\boldsymbol{x}}^{2} G(\boldsymbol{x}, \boldsymbol{z})_{j m} \overline{\overline{\mathcal{M}}}_{m i}
\end{aligned}
$$

where $\overline{\widetilde{\mathcal{M}}}:=-\breve{\mathcal{C}}+\mathcal{N}$ is a total reduced rank 2 tensor. Furthermore, in Appendix B, we show that this new tensor is symmetric and, therefore, has just 6 independent coefficients. It then directly follows that

$$
\begin{aligned}
\left(\boldsymbol{H}_{\alpha}-\boldsymbol{H}_{0}\right)(\boldsymbol{x})_{j}= & \boldsymbol{D}_{\boldsymbol{x}}^{2} G(\boldsymbol{x}, \boldsymbol{z})_{j m} \overline{\overline{\mathcal{M}}}_{m i} \boldsymbol{H}_{0}(\boldsymbol{z})_{i} \\
& +\boldsymbol{R}(\boldsymbol{x})_{j} .
\end{aligned}
$$

If the field $\boldsymbol{H}_{0}(\boldsymbol{z})$ is taken to be that obtained from a dipole positioned at $s$ with (unit) magnetic moment $\hat{v}$ then, by taking the dot product of (24) with the (unit) moment $\hat{\boldsymbol{u}}$, we have

$$
\begin{aligned}
\hat{\boldsymbol{u}} \cdot\left(\boldsymbol{H}_{\alpha}-\boldsymbol{H}_{0}\right)(\boldsymbol{x})= & \left(\boldsymbol{D}_{\boldsymbol{x}}^{2} G(\boldsymbol{x}, \boldsymbol{z}) \hat{\boldsymbol{u}}\right) \cdot \overline{(\overline{\mathcal{M}}} \\
& \left.\left(\boldsymbol{D}_{\boldsymbol{x}}^{2} G(\boldsymbol{z}, \boldsymbol{s}) \hat{\boldsymbol{v}}\right)\right)+\boldsymbol{R}(\boldsymbol{x}) .
\end{aligned}
$$

which, with $\boldsymbol{H}^{T}(\boldsymbol{z}) \quad:=\underline{\boldsymbol{D}}_{\boldsymbol{x}}^{2} G(\boldsymbol{z}, \boldsymbol{s}) \hat{\boldsymbol{u}}, \quad \boldsymbol{H}^{M}(\boldsymbol{z}) \quad:=$ $\boldsymbol{D}_{\boldsymbol{x}}^{2} G(\boldsymbol{x}, \boldsymbol{z}) \hat{\boldsymbol{v}}$ and $\mathcal{M}^{\text {ind }}:=\widetilde{\mathcal{M}}$, has the same form as the engineering prediction in (1), a fact that we will return to in Section VI.

\section{SIMPLIFIED POLARISATION TENSORS FOR CLASSES OF GEOMETRIES}

The number of independent coefficients that are required to define $\mathcal{C}$ and $\mathcal{N}$ (and hence $\check{\mathcal{C}}, \mathcal{P}, \widehat{\widehat{\mathcal{N}}}, \mathcal{M}$ and $\breve{M}$ ) for an object with either a rotational or mirror symmetry (or multiple symmetries, or both) are often fewer than those required to define a general object. To explain this, we show how the number of independent coefficients can be reduced for an object which has a uniaxial symmetry and an object which has both a uniaxial symmetry and additional mirror symmetries. We then apply similar techniques to a range of simple objects and consider the further simplification that results in the case of a spherical object.

\section{A. Polarisation tensors for objects with uniaxial symmetry}

The number of independent coefficients for an object, which has uniaxial symmetry, in a given direction, can either be determined by a counting argument or by considering the elements of the tensor that should remain invariant under a rotation. Here we apply the latter and first consider the conductivity tensor $\mathcal{P}$, which can be expressed in terms of the rank 3 tensor density $\mathcal{C}=\sum \mathcal{C}_{m s i} \hat{\boldsymbol{e}}_{m} \otimes \hat{\boldsymbol{e}}_{s} \otimes \hat{\boldsymbol{e}}_{i}$. We remark that one could also express this in terms of the rank 2 tensor $\breve{\mathcal{C}}$, but instead we apply the former and use the skew symmetry of $\mathcal{C}$. Under the transformation by an orthogonal matrix $\mathcal{R}$ the rank 3 tensor density becomes

$$
\mathcal{C}_{i j k}^{\prime}=|\mathcal{R}| \mathcal{R}_{i \ell} \mathcal{R}_{j m} \mathcal{R}_{k n} \mathcal{C}_{\ell m n}
$$

where $|\mathcal{R}|$ denotes the determinate, which is always 1 for a proper transformation. For example, for a rotation of angle $\psi$ about $\hat{\boldsymbol{e}}_{3}$ then

$$
\mathcal{R}=\left(\begin{array}{ccc}
\cos \psi & \sin \psi & 0 \\
-\sin \psi & \cos \psi & 0 \\
0 & 0 & 1
\end{array}\right)
$$

If an object has uniaxial symmetry in the $\hat{\boldsymbol{e}}_{3}$ coordinate direction then this means that the tensor $\mathcal{C}$ should be invariant under a $\psi=\pi / 2$ rotation about the $\hat{\boldsymbol{e}}_{3}$ axis $: \hat{\boldsymbol{e}}_{1} \rightarrow \hat{\boldsymbol{e}}_{2}$ and $\hat{\boldsymbol{e}}_{2} \rightarrow-\hat{\boldsymbol{e}}_{1}$. Under this transformation $\mathcal{C}^{\prime}=\mathcal{C}$ and applying (26) to $\mathcal{C}^{\prime}$ gives $\mathcal{C}^{\prime \prime}=\mathcal{C}^{\prime}=\mathcal{C}$. By considering a general $\mathcal{C}$ it follows that it's 7 independent coefficients are $\mathcal{C}_{333}, \mathcal{C}_{311}=\mathcal{C}_{322}, \mathcal{C}_{131}=\mathcal{C}_{232}, \mathcal{C}_{113}=\mathcal{C}_{223}, \mathcal{C}_{312}=-\mathcal{C}_{321}$, $\mathcal{C}_{132}=-\mathcal{C}_{231}$ and $\mathcal{C}_{123}=-\mathcal{C}_{213}$. But, we also know that $\mathcal{C}$ is skew symmetric with respect to the first two indices and this reduces the number to just 3: $\mathcal{C}_{312}=-\mathcal{C}_{321}=-\mathcal{C}_{132}=\mathcal{C}_{231}$, $\mathcal{C}_{123}=-\mathcal{C}_{213}$ and $\mathcal{C}_{311}=-\mathcal{C}_{131}=\mathcal{C}_{322}=-\mathcal{C}_{232}$.

Secondly, we consider the permeability tensor $\mathcal{N}=$ $\sum \mathcal{N}_{\ell i} \hat{\boldsymbol{e}}_{\ell} \otimes \hat{\boldsymbol{e}}_{i}$ which transforms as

$$
\mathcal{N}_{i j}^{\prime}=\mathcal{R}_{i \ell} \mathcal{R}_{j m} \mathcal{N}_{\ell m} .
$$

Then, by proceeding in a similar manner to above, for an object with uniaxial symmetry in the $\hat{e}_{3}$ direction we have $\mathcal{N}_{11}=\mathcal{N}_{22}, \mathcal{N}_{12}=-\mathcal{N}_{21}$ and $\mathcal{N}_{33}$.

Finally, we know that the total reduced tensor $\widetilde{\mathcal{M}}=-\breve{\mathcal{C}}+\mathcal{N}$ is symmetric, so that $\overline{\mathcal{M}_{12}}=\overline{\mathcal{M}_{21}}$, but rotational symmetry tell us $\underline{\mathcal{N}_{12}}=-\mathcal{N}_{21}$ and $\breve{\mathcal{C}}_{12}=-\breve{\mathcal{C}}_{21}=\mathcal{C}_{232}$ so that $\overline{\mathcal{M}}_{12}=\overline{\mathcal{M}}_{21}=-\mathcal{C}_{232}+\mathcal{N}_{12}=\mathcal{C}_{232}-\mathcal{N}_{12}=0$. Thus $\overline{\mathcal{M}}$ is diagonal in this case with just 2 independent coefficients: $\overline{\mathcal{M}}_{11}=\overline{\mathcal{M}}_{22}=\mathcal{N}_{11}-\mathcal{C}_{231}$ and $\overline{\mathcal{M}}_{33}=\mathcal{N}_{33}-\mathcal{C}_{123}$.

\section{B. Polarisation tensors for objects with both uniaxial and mirror symmetries}

The number of independent coefficients that are required to define $\mathcal{C}$ and $\mathcal{N}$ can also be reduced if the object has mirror symmetries. For an object with a mirror symmetry associated with the plane with unit normal vector $\hat{\boldsymbol{n}}$ then

$$
\mathcal{R}_{i j}=\delta_{i j}-2 \hat{n}_{i} \hat{n}_{j} .
$$

The elements of the rank 3 tensor density $\mathcal{C}$ and the rank 2 tensor $\mathcal{N}$ remain invariant under a reflection provided that the last index remains unchanged under the transformation. For an object with mirror symmetries associated with planes with normals $\hat{\boldsymbol{e}}_{1}, \hat{\boldsymbol{e}}_{2}, \hat{\boldsymbol{e}}_{3}$ this gives the 6 independent coefficients $\mathcal{C}_{231}, \mathcal{C}_{321}, \mathcal{C}_{132}, \mathcal{C}_{312}, \mathcal{C}_{123}$ and $\mathcal{C}_{213}$ for a general $\mathcal{C}$, which reduces to 3 on consideration of the skew symmetry of $\mathcal{C}$, and gives the 3 independent coefficients $\mathcal{N}_{11}, \mathcal{N}_{22}$ and $\mathcal{N}_{33}$ for $\mathcal{N}$. The total tensor $\overline{\mathcal{M}}$ is then diagonal with 3 independent coefficients: $\overline{\mathcal{M}}_{11}=\mathcal{N}_{11}-\mathcal{C}_{231}, \overline{\mathcal{M}}_{22}=\mathcal{N}_{22}-\mathcal{C}_{312}$ and $\overline{\mathcal{M}}_{33}=\mathcal{N}_{33}-\mathcal{C}_{123}$. If an object also has mirror symmetries associated with planes with normals $\hat{\boldsymbol{e}}_{1}+\hat{\boldsymbol{e}}_{2}, \hat{\boldsymbol{e}}_{1}+\hat{\boldsymbol{e}}_{3}$ and $\hat{\boldsymbol{e}}_{2}+\hat{\boldsymbol{e}}_{3}$ then there is no further change in the independent coefficients for $\mathcal{C}, \mathcal{N}$ and $\overline{\mathcal{M}}$. But, if in addition to the aforementioned mirror symmetries, the object also has the 
uniaxial symmetry described in Section V-A then there are just 2 independent coefficients for $\mathcal{C}: \mathcal{C}_{123}=-\mathcal{C}_{213}$ and $\mathcal{C}_{312}=-\mathcal{C}_{132}=\mathcal{C}_{231} \equiv-\mathcal{C}_{321} ; 2$ for $\mathcal{N}: \mathcal{N}_{11}=\mathcal{N}_{22}$ and $\mathcal{N}_{33}$; and 2 for $\overline{\mathcal{M}}: \overline{\mathcal{M}}_{11}=\overline{\mathcal{M}}_{22}=\mathcal{N}_{11}-\mathcal{C}_{231}$ and $\overline{\mathcal{M}}_{33}=\mathcal{N}_{33}-\mathcal{C}_{123}$. Such an object is a cube with a hole.

\section{Examples of symmetries in polarisation tensors}

By applying similar arguments to those described above, the entries in Table I, which lists the independent coefficients for some simple shapes, can be identified. The rotational symmetries of an object about an angle $\pi$ in a given coordinate direction, which are equivalent to an appropriate mirror symmetry, have been omitted. Note that the independent coefficients that define a sphere and a cube are the same, as are the cases of a cylinder and a cone when their axes are aligned. Furthermore, for all the simple objects in Table I, we observe that the rank 2 tensor $\overline{\overline{\mathcal{M}}}=-\check{\mathcal{C}}+\mathcal{N}$ is diagonal.

\section{Polarisation tensor for a spherical geometry}

The polarisation tensor for a spherical object, which has been obtained by Ammari et al. [26], is a further simplification of (24) where $\overline{\mathcal{M}}_{\ell i}=-\breve{\mathcal{C}}_{\ell i}+\mathcal{N}_{\ell i}=(-C+N) \delta_{\ell i}$ is scalar multiple of the identity tensor. Here, it can be shown that

$$
\begin{gathered}
N:=\frac{1}{3} \mathcal{N}_{p p}, \\
C:=\frac{1}{6} \varepsilon_{m s i} \mathcal{C}_{m s i}=\frac{\beta}{6} \varepsilon_{m s i} \int_{B} \xi_{m}\left(\theta_{s i}+\varepsilon_{s i q} \xi_{q}\right) \mathrm{d} \boldsymbol{\xi} \\
=\frac{\beta}{6} \int_{B}\left(\varepsilon_{m s i} \xi_{m} \theta_{s i}+2 \xi_{q} \xi_{q}\right) \mathrm{d} \boldsymbol{\xi} \\
=-\beta \int_{B}\left(\xi_{1} \boldsymbol{\theta}_{2} \cdot \boldsymbol{e}_{3}-\xi_{1}^{2}\right) \mathrm{d} \boldsymbol{\xi},
\end{gathered}
$$

by noting that $\int_{B} \xi_{1}^{2} \mathrm{~d} \boldsymbol{\xi}=\int_{B} \xi_{2}^{2} \mathrm{~d} \boldsymbol{\xi}=\int_{B} \xi_{3}^{2} \mathrm{~d} \boldsymbol{\xi}$ for a sphere and using integration by parts to show that $\int_{B} \xi_{1} \boldsymbol{\theta}_{3} \cdot \boldsymbol{e}_{2} \mathrm{~d} \boldsymbol{\xi}=$ $-\int_{B} \xi_{1} \boldsymbol{\theta}_{2} \cdot \boldsymbol{e}_{3} \mathrm{~d} \boldsymbol{\xi}$.

Using the analytical solution [14] for the eddy currents generated in a conducting (magnetic) sphere of radius $\alpha$ with conductivity $\sigma_{*}$, permeability $\mu_{*}$ at angular frequency $\omega$, when placed in a uniform field $\boldsymbol{H}_{0}$, we can show that the form of the perturbed field is identical to (24), if we set $\boldsymbol{R}=\mathbf{0}$ and $\overline{\mathcal{M}}_{\ell i}=(-C+N) \delta_{\ell i}$. It can also be identified, that

$$
\begin{aligned}
\overline{-C+N} & =2 \pi . \\
& \frac{\left(\left(2 \mu_{*}+\mu_{0}\right) v I_{-1 / 2}-\left(\mu_{0}\left(1+v^{2}\right)+2 \mu_{*}\right) I_{1 / 2}\right)}{\left(\mu_{*}-\mu_{0}\right) I_{-1 / 2}+\left(\mu_{0}\left(1+v^{2}\right)-\mu_{*}\right) I_{1 / 2}},
\end{aligned}
$$

where $v=\sqrt{\mathrm{i} \sigma \mu_{*} \omega} \alpha, I_{1 / 2}(v)=\sqrt{\frac{2}{\pi v}} \sinh v$ and $I_{-1 / 2}(v)=$ $\sqrt{\frac{2}{\pi v}} \cosh v$. The overline indicates the complex conjugate, which appears due to the $e^{\mathrm{i} \omega t}$ time variation in [14] rather than the $e^{-\mathrm{i} \omega t}$ assumed here.

An analytical solution [16] is also available for case where the same sphere is now illuminated by an incident field generated by a circular coil of radius $\gamma$ carrying an alternating current $I$. For a coil centred at $s$ the incident magnetic field at position $z$ can be described in terms of a magnetic dipole in the form

$$
\boldsymbol{H}_{0}(\boldsymbol{z})=\boldsymbol{D}_{\boldsymbol{x}}^{2} G(\boldsymbol{z}, \boldsymbol{s}) \boldsymbol{m},
$$

provided that the length of coil, $L=2 \pi \gamma$, is small compared to the distance from the coil to the object, $|z-s|$. In the above, $\boldsymbol{m}$ is the magnetic dipole moment of the current source, which, for a circular coil, has $|\boldsymbol{m}|=I \pi \gamma^{2}$ [31]. If the coil is chosen to lie in the $\left(\hat{\boldsymbol{e}}_{1}, \hat{\boldsymbol{e}}_{2}\right)$ plane then $\boldsymbol{m}=I \pi \gamma^{2} \hat{\boldsymbol{e}}_{3}$ and

$$
\boldsymbol{H}_{0}(\boldsymbol{z})=I \pi \gamma^{2} \boldsymbol{D}_{\boldsymbol{x}}^{2} G(\boldsymbol{z}, \boldsymbol{s}) \hat{\boldsymbol{e}}_{3} .
$$

Furthermore, we can show that the leading order term for $\left(\boldsymbol{H}_{\alpha}-\boldsymbol{H}_{0}\right)(\boldsymbol{x})$ that Wait [16] obtains is identical to that described by (24).

\section{RECIPROCITY IMPLICATIONS}

In the introduction we remarked how the engineering prediction of $V^{i n d} \approx \boldsymbol{H}^{M} \cdot\left(\mathcal{M}^{i n d} \boldsymbol{H}^{T}\right)$ for a conducting object, where $\mathcal{M}^{\text {ind }}$ is a symmetric rank 2 tensor, sits comfortably with the basic idea of reciprocity that states that the response of a system is unchanged when the transmitter and receiver are interchanged. Let us now formalise this more precisely.

Recall the Lorentz reciprocity principal, which is usually formulated for the time harmonic equations, in the form [13], [32]

$$
\nabla \cdot\left(\boldsymbol{E}^{a} \times \boldsymbol{H}^{b}-\boldsymbol{E}^{b} \times \boldsymbol{H}^{a}\right)=\boldsymbol{J}_{0}^{a} \cdot \boldsymbol{E}^{b}-\boldsymbol{J}_{0}^{b} \cdot \boldsymbol{E}^{a},
$$

or, by integrating over $\mathbb{R}^{3}$ and using the far field behaviour of the fields, as

$$
\int_{\mathbb{R}^{3}} \boldsymbol{J}_{0}^{a} \cdot \boldsymbol{E}^{b} \mathrm{~d} \boldsymbol{x}=\int_{\mathbb{R}^{3}} \boldsymbol{J}_{0}^{b} \cdot \boldsymbol{E}^{a} \mathrm{~d} \boldsymbol{x} .
$$

It follows from this result that the response is unchanged when the transmitter and receiver are interchanged. Furthermore, if the derivation is repeated for the eddy current model, the result (33) is again obtained. Then, if we follow [32], and assume the current sources $a, b$ to have a small support and to be located at $s$ and $t$, respectively, then the first term in a Taylor series of expansion of the fields $\boldsymbol{E}^{a}$ and $\boldsymbol{E}^{b}$ about the centre of the current source is

$$
\boldsymbol{E}^{b}(\boldsymbol{s}) \cdot \boldsymbol{p}^{a} \approx \boldsymbol{E}^{a}(\boldsymbol{t}) \cdot \boldsymbol{p}^{b},
$$

where $\boldsymbol{p}^{a}$ is the electric dipole moment of the current source $a$. It is important to note that this is only the first term in the Taylor's series expansion, including the next term leads to

$$
\begin{aligned}
\boldsymbol{E}^{b}(\boldsymbol{s}) \cdot \boldsymbol{p}^{a}+2 \nabla^{s} \boldsymbol{E}^{b}(\boldsymbol{s}): \mathcal{R}^{a}+\boldsymbol{B}^{b}(\boldsymbol{s}) \cdot \boldsymbol{m}^{a} \approx \\
\boldsymbol{E}^{a}(\boldsymbol{t}) \cdot \boldsymbol{p}^{b}+2 \nabla^{s} \boldsymbol{E}^{a}(\boldsymbol{t}): \mathcal{R}^{b}+\boldsymbol{B}^{a}(\boldsymbol{t}) \cdot \boldsymbol{m}^{b},
\end{aligned}
$$

where $\mathcal{R}^{a}$ is a quadrupole moment of the current source $a$, $\boldsymbol{m}^{a}$ the magnetic moment of the same current source [32] and exact reciprocity is expected if all the terms in the Taylor series expansion are considered.

For the eddy current problem described in this work and coils located in free space that can be idealised as dipoles with a magnetic moment, only, reciprocity implies that $\boldsymbol{m}^{a}$. 
TABLE I

NON-ZERO INDEPENDENT COEFFICIENTS THAT ARE REQUIRED TO REPRESENT $\mathcal{C}$ AND $\mathcal{N}$ FOR A RANGE OF SIMPLE OBJECTS.

\begin{tabular}{|c|c|c|c|c|}
\hline $\begin{array}{l}\text { Object Shape } \\
\text { Object Shape }\end{array}$ & $\begin{array}{c}\text { Rotational } \\
\text { Symmetries }\end{array}$ & $\begin{array}{c}\text { Mirror } \\
\text { Symmetries } \\
\end{array}$ & $\begin{array}{c}\text { Independent } \\
\text { Coefficients in } \mathcal{C}\end{array}$ & $\begin{array}{c}\text { Independent } \\
\text { Coefficients in } \mathcal{N}\end{array}$ \\
\hline Sphere & Isotropic & $\begin{array}{l}\text { Infinite number } \\
\text { of planes }\end{array}$ & $\begin{array}{c}\mathcal{C}_{123}=-\mathcal{C}_{132}=-\mathcal{C}_{213}= \\
\mathcal{C}_{231}=\mathcal{C}_{312}=-\mathcal{C}_{321}\end{array}$ & $\mathcal{N}_{11}=\mathcal{N}_{22}=\mathcal{N}_{33}$ \\
\hline $\begin{array}{c}\text { Cube: } \\
\text { Aligned with axes }\end{array}$ & $\begin{array}{l}\text { Uniaxial about } \\
\hat{\boldsymbol{e}}_{1}, \hat{\boldsymbol{e}}_{2}, \hat{\boldsymbol{e}}_{3}\end{array}$ & $\begin{array}{c}\text { Planes with normals } \\
\hat{\boldsymbol{e}}_{1}, \hat{\boldsymbol{e}}_{2}, \hat{\boldsymbol{e}}_{3} \\
\left(\hat{\boldsymbol{e}}_{1}+\hat{\boldsymbol{e}}_{2}\right) / \sqrt{2} \\
\left(\hat{\boldsymbol{e}}_{1}+\hat{\boldsymbol{e}}_{3}\right) / \sqrt{2} \\
\left(\hat{\boldsymbol{e}}_{2}+\hat{\boldsymbol{e}}_{3}\right) / \sqrt{2} \\
\end{array}$ & $\begin{array}{c}\mathcal{C}_{123}=-\mathcal{C}_{132}=-\mathcal{C}_{213}= \\
\mathcal{C}_{231}=\mathcal{C}_{312}=-\mathcal{C}_{321}\end{array}$ & $\mathcal{N}_{11}=\mathcal{N}_{22}=\mathcal{N}_{33}$ \\
\hline $\begin{array}{c}\text { Block: } \\
\left(-\frac{w}{2},-\frac{d}{2},-\frac{h}{2}\right) \times \\
\left(\frac{w}{2}, \frac{d}{2}, \frac{h}{2}\right) \\
\text { Aligned with axes }\end{array}$ & None & $\begin{array}{c}\text { Planes with normals } \\
\hat{\boldsymbol{e}}_{1}, \hat{\boldsymbol{e}}_{2}, \hat{\boldsymbol{e}}_{3}, \\
\left(d \boldsymbol{e}_{1}+w \boldsymbol{e}_{2}\right) / \sqrt{d^{2}+w^{2}} \\
\left(w \boldsymbol{e}_{3}+h \boldsymbol{e}_{1}\right) / \sqrt{w^{2}+h^{2}} \\
\left(h \boldsymbol{e}_{2}+d \boldsymbol{e}_{3}\right) / \sqrt{d^{2}+h^{2}}\end{array}$ & $\begin{array}{l}\mathcal{C}_{123}=-\mathcal{C}_{213} \\
\mathcal{C}_{321}=-\mathcal{C}_{231} \\
\mathcal{C}_{132}=-\mathcal{C}_{312}\end{array}$ & $\begin{array}{l}\mathcal{N}_{11} \\
\mathcal{N}_{22} \\
\mathcal{N}_{33}\end{array}$ \\
\hline $\begin{array}{l}\text { Cone: } \\
\text { Axis aligned with } \hat{\boldsymbol{e}}_{1}\end{array}$ & $\begin{array}{c}\text { Rotationally } \\
\text { invariant about } \\
\hat{\boldsymbol{e}}_{1}\end{array}$ & $\begin{array}{c}\text { Planes with normals } \\
\hat{\boldsymbol{e}}_{2}, \hat{\boldsymbol{e}}_{3} \\
\text { any plane } \perp \text { base \& } \\
\text { passing through the vertex }\end{array}$ & $\begin{array}{c}\mathcal{C}_{123}=-\mathcal{C}_{132}=-\mathcal{C}_{213}=\mathcal{C}_{312} \\
\mathcal{C}_{231}=-\mathcal{C}_{321}\end{array}$ & $\begin{array}{c}\mathcal{N}_{11} \\
\mathcal{N}_{22}=\mathcal{N}_{33}\end{array}$ \\
\hline $\begin{array}{c}\text { Cylinder: } \\
\text { Axis aligned with } \hat{e}_{1}\end{array}$ & $\begin{array}{c}\text { Rotationally } \\
\text { invariant about } \\
\hat{e}_{1}\end{array}$ & $\begin{array}{c}\text { Planes with normals } \\
\hat{\boldsymbol{e}}_{1}, \hat{\boldsymbol{e}}_{2}, \hat{\boldsymbol{e}}_{3} \\
\text { any plane } \perp \text { base \& } \\
\text { parallel to } \hat{\boldsymbol{e}}_{1}\end{array}$ & $\begin{array}{c}\mathcal{C}_{123}=-\mathcal{C}_{132}=-\mathcal{C}_{213}=\mathcal{C}_{312} \\
\mathcal{C}_{231}=-\mathcal{C}_{321}\end{array}$ & $\begin{array}{c}\mathcal{N}_{11} \\
\mathcal{N}_{22}=\mathcal{N}_{33}\end{array}$ \\
\hline $\begin{array}{l}\text { Cube with hole: } \\
\text { Hole aligned with axis } \hat{e}_{3}\end{array}$ & $\begin{array}{c}\text { Uniaxial about } \\
\hat{e}_{3}\end{array}$ & $\begin{array}{c}\text { Planes with normals } \\
\hat{\boldsymbol{e}}_{1}, \hat{\boldsymbol{e}}_{2}, \hat{\boldsymbol{e}}_{3} \\
\left(\hat{\boldsymbol{e}}_{1}+\hat{\boldsymbol{e}}_{2}\right) / \sqrt{2} \\
\left(\hat{\boldsymbol{e}}_{1}+\hat{\boldsymbol{e}}_{3}\right) / \sqrt{2} \\
\left(\hat{\boldsymbol{e}}_{2}+\hat{\boldsymbol{e}}_{3}\right) / \sqrt{2}\end{array}$ & $\begin{array}{c}\mathcal{C}_{123}=-\mathcal{C}_{213} \\
\mathcal{C}_{132}=-\mathcal{C}_{231}=\mathcal{C}_{321}=-\mathcal{C}_{312}\end{array}$ & $\begin{array}{c}\mathcal{N}_{11}=\mathcal{N}_{22} \\
\mathcal{N}_{33}\end{array}$ \\
\hline $\begin{array}{c}\text { Ellipsoid: } \\
\text { Major axis aligned with } \hat{\boldsymbol{e}}_{3}\end{array}$ & $\begin{array}{c}\text { Rotationally } \\
\text { invariant about } \\
\hat{\boldsymbol{e}}_{3}\end{array}$ & $\begin{array}{c}\text { Planes with normals } \\
\hat{\boldsymbol{e}}_{1}, \hat{\boldsymbol{e}}_{2}, \hat{\boldsymbol{e}}_{3} \\
\text { any plane parallel } \\
\text { to } \hat{\boldsymbol{e}}_{3}\end{array}$ & $\begin{array}{c}\mathcal{C}_{123}=-\mathcal{C}_{213} \\
\mathcal{C}_{132}=-\mathcal{C}_{231}=\mathcal{C}_{321}=-\mathcal{C}_{312}\end{array}$ & $\begin{array}{c}\mathcal{N}_{11}=\mathcal{N}_{22} \\
\mathcal{N}_{33}\end{array}$ \\
\hline
\end{tabular}

$\boldsymbol{H}_{\alpha}^{b}(\boldsymbol{s}) \approx \boldsymbol{m}^{b} \cdot \boldsymbol{H}_{\alpha}^{a}(\boldsymbol{t})$ i.e. the result is the same if $\boldsymbol{s}$ and $\boldsymbol{t}$ and $\boldsymbol{m}^{a}$ and $\boldsymbol{m}^{b}$ are interchanged. Considering (13), we have

$$
\begin{aligned}
\boldsymbol{m}_{j}^{a} \boldsymbol{H}_{\alpha}^{b}(\boldsymbol{s})_{j}= & \boldsymbol{m}_{j}^{a} \boldsymbol{H}_{0}^{b}(\boldsymbol{s})_{j}+\boldsymbol{m}_{j}^{a} \boldsymbol{D}_{\boldsymbol{x}}^{2} G(\boldsymbol{s}, \boldsymbol{z})_{\ell m} \mathcal{M}_{\ell m j i} \boldsymbol{H}_{0}^{b}(\boldsymbol{z})_{i} \\
& +\boldsymbol{m}_{j}^{a} \boldsymbol{R}^{b}(\boldsymbol{s})_{j}, \\
\boldsymbol{m}_{j}^{b} \boldsymbol{H}_{\alpha}^{a}(\boldsymbol{t})_{j}= & \boldsymbol{m}_{j}^{b} \boldsymbol{H}_{0}^{a}(\boldsymbol{t})_{j}+\boldsymbol{m}_{j}^{b} \boldsymbol{D}_{\boldsymbol{x}}^{2} G(\boldsymbol{t}, \boldsymbol{z})_{\ell m} \mathcal{M}_{\ell m j i} \boldsymbol{H}_{0}^{a}(\boldsymbol{z})_{i} \\
& +\boldsymbol{m}_{j}^{b} \boldsymbol{R}^{a}(\boldsymbol{t})_{j} .
\end{aligned}
$$

In the case considered, $\boldsymbol{H}_{0}^{b}(\boldsymbol{s})=\boldsymbol{D}_{\boldsymbol{x}}^{2} G(\boldsymbol{s}, \boldsymbol{t}) \boldsymbol{m}^{b}$ and $\boldsymbol{H}_{0}^{a}(\boldsymbol{t})=\boldsymbol{D}_{\boldsymbol{x}}^{2} G(\boldsymbol{t}, \boldsymbol{s}) \boldsymbol{m}^{a}$, thus, from the symmetry of $\boldsymbol{D}_{\boldsymbol{x}}^{2} G(\boldsymbol{s}, \boldsymbol{t})$, we have $\boldsymbol{m}^{a} \cdot \boldsymbol{H}_{0}^{b}(\boldsymbol{s})=\boldsymbol{m}^{b} \cdot \boldsymbol{H}_{0}^{a}(\boldsymbol{t})$. Indeed, we can easily obtain that

$$
\begin{aligned}
\boldsymbol{m}_{j}^{a} & \boldsymbol{H}_{\alpha}^{b}(\boldsymbol{s})_{j}-\boldsymbol{m}_{j}^{b} \boldsymbol{H}_{\alpha}^{a}(\boldsymbol{t})_{j}=\Delta\left(\boldsymbol{m}^{a}, \boldsymbol{m}^{b}, \boldsymbol{s}, \boldsymbol{t}\right) \\
& +\boldsymbol{m}_{j}^{a} \boldsymbol{D}_{\boldsymbol{x}}^{2} G(\boldsymbol{s}, \boldsymbol{z})_{\ell m} \mathcal{M}_{\ell m j i} \boldsymbol{D}_{\boldsymbol{x}}^{2} G(\boldsymbol{z}, \boldsymbol{t})_{i p} \boldsymbol{m}_{p}^{b} \\
& -\boldsymbol{m}_{j}^{b} \boldsymbol{D}_{\boldsymbol{x}}^{2} G(\boldsymbol{t}, \boldsymbol{z})_{\ell m} \mathcal{M}_{\ell m j i} \boldsymbol{D}_{\boldsymbol{x}}^{2} G(\boldsymbol{z}, \boldsymbol{s})_{i p} \boldsymbol{m}_{p}^{a},
\end{aligned}
$$

where $\Delta:=\boldsymbol{m}^{a} \cdot \boldsymbol{R}^{b}(\boldsymbol{s})-\boldsymbol{m}^{b} \cdot \boldsymbol{R}^{a}(\boldsymbol{t})$ and

$$
\begin{aligned}
|\Delta| & \leqslant\left|\boldsymbol{m}^{a}\right|\left|\boldsymbol{R}^{b}(s)\right|+\left|\boldsymbol{m}^{b}\right|\left|\boldsymbol{R}^{a}(\boldsymbol{t})\right| \\
& \leqslant C \alpha^{4}\left(\left|\boldsymbol{m}^{a}\right|\left\|\boldsymbol{H}_{0}^{b}\right\|_{W^{2, \infty}\left(B_{\alpha}\right)}+\left|\boldsymbol{m}^{b}\right|\left\|\boldsymbol{H}_{0}^{a}\right\|_{W^{2, \infty}\left(B_{\alpha}\right)}\right),
\end{aligned}
$$

thus (36) is an asymptotic expansion for $\boldsymbol{m}^{a} \cdot \boldsymbol{H}_{\alpha}^{b}(\boldsymbol{s})-\boldsymbol{m}^{b}$. $\boldsymbol{H}_{\alpha}^{a}(\boldsymbol{t})$ as $\alpha \rightarrow 0$ with $\Delta=O\left(\alpha^{4}\right)$. Reciprocity then implies that

$$
\begin{aligned}
& \boldsymbol{m}_{j}^{a} \boldsymbol{D}_{\boldsymbol{x}}^{2} G(\boldsymbol{s}, \boldsymbol{z})_{\ell m} \mathcal{M}_{\ell m j i} \boldsymbol{D}_{\boldsymbol{x}}^{2} G(\boldsymbol{z}, \boldsymbol{t})_{i p} \boldsymbol{m}_{p}^{b} \\
& \quad \approx \boldsymbol{m}_{j}^{b} \boldsymbol{D}_{\boldsymbol{x}}^{2} G(\boldsymbol{t}, \boldsymbol{z})_{\ell m} \mathcal{M}_{\ell m j i} \boldsymbol{D}_{\boldsymbol{x}}^{2} G(\boldsymbol{z}, \boldsymbol{s})_{i p} \boldsymbol{m}_{p}^{a},
\end{aligned}
$$

upto an error term $\Delta$, or alternatively in terms of the symmetric rank 2 tensor $\overline{\mathcal{M}}$, by using (23), that

$$
\begin{aligned}
& \boldsymbol{m}_{j}^{a} \boldsymbol{D}_{\boldsymbol{x}}^{2} G(\boldsymbol{s}, \boldsymbol{z})_{j m} \overline{\overline{\mathcal{M}}}_{m i} \boldsymbol{D}_{\boldsymbol{x}}^{2} G(\boldsymbol{z}, \boldsymbol{t})_{i p} \boldsymbol{m}_{p}^{b} \\
& \quad \approx \boldsymbol{m}_{j}^{b} \boldsymbol{D}_{\boldsymbol{x}}^{2} G(\boldsymbol{t}, \boldsymbol{z})_{j m} \overline{\overline{\mathcal{M}}}_{m i} \boldsymbol{D}_{\boldsymbol{x}}^{2} G(\boldsymbol{z}, \boldsymbol{s})_{i p} \boldsymbol{m}_{p}^{a} .
\end{aligned}
$$

In light of (33), if one constructs a suitable $\boldsymbol{J}_{0}^{b}$, which has non-zero support on the measurement coil and is such that the resulting field $\boldsymbol{H}_{0}^{b}$ can be idealised as a magnetic dipole, the induced voltage, $V^{i n d}$, as a result of the perturbation caused by the presence of a general conducting object, is

$$
\begin{aligned}
V^{i n d} & \approx \boldsymbol{m}_{j}^{b} \boldsymbol{D}_{\boldsymbol{x}}^{2} G(\boldsymbol{t}, \boldsymbol{z})_{j m} \overline{\overline{\mathcal{M}}}_{m i} \boldsymbol{D}_{\boldsymbol{x}}^{2} G(\boldsymbol{z}, \boldsymbol{s})_{i p} \boldsymbol{m}_{p}^{a} \\
& \approx \boldsymbol{H}_{0}^{b}(\boldsymbol{z}) \cdot\left(\overline{\overline{\mathcal{M}}} \boldsymbol{H}_{0}^{a}(\boldsymbol{z})\right) .
\end{aligned}
$$


which, with $a=M, b=T$ and $\overline{\overline{\mathcal{M}}}=\mathcal{M}^{\text {ind }}$, agrees with the prediction in (1).

\section{Determining $\overline{\overline{\mathcal{M}}}$ From Field MEASUREMEnts}

In the next section we describe a numerical approach for computing $\boldsymbol{\theta}_{i}, i=1,2,3$, which can be used for the accurate calculation of the polarisation tensors. However, there may also be situations (e.g. as part of an inverse algorithm or an experimental validation proceedure) where the independent coefficients that define the polarisation tensors should be determined from field measurements of $\left(\boldsymbol{H}_{\alpha}-\boldsymbol{H}_{0}\right)(\boldsymbol{x})$.

For a conducting object we have found that a set of 9 independent coefficients are sufficient for describing the tensor $\mathcal{P}$. If the object is magnetic a further 9 independent coefficients are needed for describing the tensor $\mathcal{N}$. But, by using (24), the perturbed field can be expressed in terms of the symmetric rank 2 tensor $\overline{\mathcal{M}}$, which has only 6 independent coefficients. Let $u_{i}$ denote the $i$ th unknown independent coefficient of the tensor(s) and $m_{i}$ denote the $i$ th measurement of $\hat{\boldsymbol{q}}^{(i)} \cdot\left(\boldsymbol{H}_{\alpha}-\boldsymbol{H}_{0}\right)\left(\boldsymbol{x}^{(i)}\right)$, where it is important that the number of measurements exceeds the number of independent coefficients and that the measurements are taken at different distances $\boldsymbol{x}^{(i)}$ and for different orientations of $\hat{\boldsymbol{q}}^{(i)}$. Then, by expressing the asymptotic expansion for $\hat{\boldsymbol{q}}^{(i)} \cdot\left(\boldsymbol{H}_{\alpha}-\boldsymbol{H}_{0}\right)\left(\boldsymbol{x}^{(i)}\right)$ in terms of the coefficients $A_{i j}$ of the $j$ th independent coefficient $u_{j}$ a set of over determined error equations can be built and solved by applying standard least squares techniques.

\section{VIII. $h p$-Finite Element Methodology for the COMPUTATIONS OF $\mathcal{M}$}

The transmission problem (10) has similarities to the $\boldsymbol{A}$ based formulation of eddy current problems e.g. [28]. We therefore advocate that the regularised formulation previously developed for eddy current problems on multiply connected domains be adapted for the solution of (10). For this purpose, we truncate the otherwise unbounded domain $B^{c}$ at a finite distance from the object and create the finite domain $\Omega=\tilde{B}^{c} \cup B$ and on the truncated boundary $\partial \Omega$ we impose $\nabla_{\boldsymbol{\xi}} \times \boldsymbol{\theta}_{i} \times \hat{\boldsymbol{n}}=\mathbf{0}$.

\section{A. Regularised formulation}

Let $\boldsymbol{\vartheta}_{i}=\overline{\boldsymbol{\theta}}_{i}$. The transmission problem for $\boldsymbol{\vartheta}_{i}$ on the finite (computational) domain can then be written in the form

$$
\begin{array}{rlrl}
\nabla_{\boldsymbol{\xi}} \times \tilde{\mu}_{r}^{-1} \nabla_{\boldsymbol{\xi}} \times \boldsymbol{\vartheta}_{i}+ & \\
\mathrm{i} \mu_{0} \omega \sigma \alpha^{2} \boldsymbol{\vartheta}_{i}=-\mathrm{i} \mu_{0} \omega \sigma \alpha^{2} \hat{\boldsymbol{e}}_{i} \times \boldsymbol{\xi} & & \text { in } B \cup \tilde{B}^{c}, \\
\nabla_{\boldsymbol{\xi}} \cdot \boldsymbol{\vartheta}_{i} & =0 & & \text { in } \tilde{B}^{c}, \\
{\left[\boldsymbol{\vartheta}_{i} \times \hat{\boldsymbol{n}}_{\Gamma}\right.} & =\mathbf{0} & & \text { on } \Gamma, \\
{\left[\tilde{\mu}_{r}^{-1} \nabla_{\boldsymbol{\xi}} \times \boldsymbol{\vartheta}_{i} \times \hat{\boldsymbol{n}}\right]_{\Gamma}=} & \\
-2\left[\tilde{\mu}_{r}^{-1}\right]_{\Gamma} \hat{\boldsymbol{e}}_{i} \times \hat{\boldsymbol{n}} & & \text { on } \Gamma, \\
\nabla_{\boldsymbol{\xi}} \times \boldsymbol{\vartheta}_{i} \times \hat{\boldsymbol{n}}=\mathbf{0} & \text { on } \partial \Omega,
\end{array}
$$

where, in the above, $\tilde{\mu}_{r}:=\mu / \mu_{0}$ such that $\tilde{\mu}_{r}=\mu_{r}=\mu_{*} / \mu_{0}$ in $B$ and $\tilde{\mu}_{r}=1$ in $\tilde{B}^{c}$.
In a weak sense, the condition $\nabla_{\boldsymbol{\xi}} \cdot \boldsymbol{\vartheta}_{i}=0$ is equivalent to $\boldsymbol{\vartheta}_{i}$ being orthogonal to all gradients (continuously extended by zero onto $\Omega$ ) [28]. This leads to the mixed problem: Find $\boldsymbol{\vartheta}_{i} \in \boldsymbol{H}(\operatorname{curl}, \Omega)$ and $\Phi \in H_{0}^{1}\left(\tilde{B}^{c}\right)$ such that

$$
\begin{aligned}
& \left(\tilde{\mu}_{r}^{-1} \nabla_{\boldsymbol{\xi}} \times \boldsymbol{\vartheta}_{i}, \nabla_{\boldsymbol{\xi}} \times \boldsymbol{v}\right)_{\Omega}+\left(\kappa \boldsymbol{\vartheta}_{i}, \boldsymbol{v}\right)_{\Omega}+\left(\nabla_{\boldsymbol{\xi}} \Phi, \boldsymbol{v}\right)_{\tilde{B}^{c}}= \\
& \quad-\left(\kappa \hat{\boldsymbol{e}}_{i} \times \boldsymbol{\xi}, \boldsymbol{v}\right)_{\Omega}-2 \int_{\Gamma}\left[\tilde{\mu}_{r}^{-1}\right] \hat{\boldsymbol{e}}_{i} \times \hat{\boldsymbol{n}} \cdot \overline{\boldsymbol{v}} \mathrm{d} \boldsymbol{\xi} \\
& \quad\left(\boldsymbol{\vartheta}_{i}, \nabla_{\boldsymbol{\xi}} \Psi\right)_{\tilde{B}^{c}}=0
\end{aligned}
$$

for all $\boldsymbol{v} \in \boldsymbol{H}(\operatorname{curl}, \Omega)$ and $\Psi \in H^{1}\left(\tilde{B}^{c}\right)$ where $\Phi$ is a Lagrange multiplier used to enforce $\nabla_{\boldsymbol{\xi}} \cdot \boldsymbol{\vartheta}_{i}=0$, in $\tilde{B}^{c}$ in a weak sense. In the above

$$
\kappa=\left\{\begin{array}{ll}
\mathrm{i} \mu_{0} \omega \sigma \alpha^{2} & \text { in } B \\
0 & \text { in } \tilde{B}^{c}
\end{array} .\right.
$$

Following [28], [33]-[36] we introduce the perturbed problem: Let $\tau>0$ be a small perturbation parameter, then: find $\boldsymbol{\vartheta}_{i}^{\tau} \in$ $\boldsymbol{H}(\operatorname{curl}, \Omega)$ such that

$$
\begin{aligned}
\left(\tilde{\mu}_{r}^{-1} \nabla_{\boldsymbol{\xi}}\right. & \left.\times \boldsymbol{\vartheta}_{i}^{\tau}, \nabla_{\boldsymbol{\xi}} \times \boldsymbol{v}\right)_{\Omega}+\left(\tilde{\kappa} \boldsymbol{\vartheta}_{i}^{\tau}, \boldsymbol{v}\right)_{\Omega}= \\
& -\left(\kappa \hat{\boldsymbol{e}}_{i} \times \boldsymbol{\xi}, \boldsymbol{v}\right)_{\Omega}-2 \int_{\Gamma}\left[\tilde{\mu}_{r}^{-1}\right] \hat{\boldsymbol{e}}_{i} \times \hat{\boldsymbol{n}} \cdot \overline{\boldsymbol{v}} \mathrm{d} \boldsymbol{\xi}
\end{aligned}
$$

for all $\boldsymbol{v} \in \boldsymbol{H}(\operatorname{curl}, \Omega)$ where

$$
\tilde{\kappa}=\left\{\begin{array}{ll}
\mathrm{i} \mu_{0} \omega \sigma \alpha^{2} & \text { in } B \\
\tau & \text { in } \tilde{B}^{c}
\end{array} .\right.
$$

The previous analysis in [33], [36] carries over to (40). For simplicity, treating only the case of $\mu_{*}=\mu_{0}$, there holds $\left\|\boldsymbol{\vartheta}_{i}-\boldsymbol{\vartheta}_{i}^{\tau}\right\|_{\boldsymbol{H}(\operatorname{curl}, \Omega)} \leqslant C \tau\left\|\kappa \hat{\boldsymbol{e}}_{i} \times \boldsymbol{\xi}\right\|_{\boldsymbol{H}_{(\operatorname{curl}, \Omega) *}}$ where the constant $C$ is independent of $\tau$ and $\boldsymbol{\vartheta}_{i}$ is the solution to (39) and $\boldsymbol{H}(\operatorname{curl}, \Omega) *$ denotes the dual space.

\section{B. Discrete approximation}

In this work we use the basis functions of Zaglmayr and Schöberl [35], [36] and we recall that for a tetrahedral triangulation consisting of vertices $\mathcal{V}_{h}$, edges $\mathcal{E}_{h}$, faces $\mathcal{F}_{h}$ and cells $\mathcal{T}_{h}$ their hierarchic $\boldsymbol{H}$ (curl) and $H^{1}(\Omega)$ conforming finite element basis can be expressed in terms of the splitting

$$
\begin{aligned}
V_{h, p}:= & V_{h}^{\mathcal{N}_{0}} \oplus \sum_{E \in \mathcal{E}_{h}} \nabla W_{p+1}^{E} \oplus \sum_{F \in \mathcal{F}_{h}} \nabla W_{p+1}^{F} \oplus \sum_{F \in \mathcal{F}_{h}} \tilde{V}_{p}^{F} \oplus \\
& \sum_{I \in \mathcal{T}_{h}} \nabla W_{p+1}^{I} \oplus \sum_{I \in \mathcal{T}_{h}} \widetilde{V}_{p}^{I} \subset \boldsymbol{H}(\operatorname{curl}, \Omega),
\end{aligned}
$$

and

$$
\begin{aligned}
W_{h, p+1}:= & W_{h, 1} \oplus \sum_{E \in \mathcal{E}_{h}} W_{p+1}^{E} \oplus \sum_{F \in \mathcal{F}_{h}} W_{p+1}^{F}+\sum_{I \in \mathcal{T}_{h}} W_{p+1}^{I} \\
& \subset H^{1}(\Omega) .
\end{aligned}
$$

In the above $V_{h}^{\mathcal{N}_{0}}$ and $W_{h, 1}$ denotes the set of lowest order Nédélec (edge element) basis functions and the standard lowest order hat functions, respectively, the former being associated with the edges of the element and the latter with the vertices of the element. The extension to arbitrary high polynomial degree order consists of the enrichment of the finite element space through the addition of higher order edge, face and interior based basis functions, $W_{p+1}^{E}, W_{p+1}^{F}$ and $W_{p+1}^{I}$, respectively, 
for $H^{1}$ and the addition of higher order edge, face and interior functions for $\boldsymbol{H}$ (curl), where, in this case, the higher order edge and some of the higher order face and interior functions are constructed from the gradients of their $H^{1}$ conforming counterparts.

Following [28], we can use the special construction of these basis functions to skip the gradient functions in $\tilde{B}^{c}$ and define a reduced $\boldsymbol{H}$ (curl) conforming space as

$$
\begin{aligned}
V_{h, p}^{\mathrm{red}}:= & V_{h}^{\mathcal{N}_{0}} \oplus \sum_{E \in \mathcal{E}_{h}^{B}} \nabla W_{p+1}^{E} \oplus \sum_{F \in \mathcal{F}_{h}^{B}} \nabla W_{p+1}^{F} \oplus \sum_{F \in \mathcal{F}_{h}} \tilde{V}_{p}^{F} \oplus \\
& \sum_{I \in \mathcal{T}_{h}^{B}} \nabla W_{p+1}^{I} \oplus \sum_{I \in \mathcal{T}_{h}} \tilde{V}_{p}^{I},
\end{aligned}
$$

where the superscript $B$ on $\mathcal{E}_{h}, \mathcal{F}_{h}$ and $\mathcal{T}_{h}$ is used to denote those edges, faces and cells associated with subdomain $B$. It then follows that the approximate ungauged weak formulation is: Find $\boldsymbol{\vartheta}_{h p}^{i} \in V_{h, p}^{\text {red }} \cap H(\operatorname{curl}, \Omega)$ such that

$$
\begin{array}{r}
\left(\tilde{\mu}_{r}^{-1} \nabla_{\boldsymbol{\xi}} \times \boldsymbol{\vartheta}_{h p}^{i}, \nabla_{\boldsymbol{\xi}} \times \boldsymbol{v}_{h p}\right)_{\Omega}+\left(\kappa \boldsymbol{\vartheta}_{h p}^{i}, \boldsymbol{v}_{h p}\right)_{\Omega}=- \\
\left(\kappa \hat{\boldsymbol{e}}_{i} \times \boldsymbol{\xi}, \boldsymbol{v}_{h p}\right)_{\Omega}-2 \int_{\Gamma}\left[\tilde{\mu}_{r}^{-1}\right] \hat{\boldsymbol{e}}_{i} \times \hat{\boldsymbol{n}} \cdot \overline{\boldsymbol{v}}_{h p} \mathrm{~d} \boldsymbol{\xi},
\end{array}
$$

$\forall \boldsymbol{v}_{h p} \in V_{h, p}^{\text {red }} \cap H(\operatorname{curl}, \Omega)$, where the solveability of (42) is ensured by gauging the low-order gradients $\nabla W_{h, 1} \subset V_{h}^{\mathcal{N}_{0}}$ through the addition of an appropriate regularisation term [28]. The structure of the left hand side of (42) is analogous to the gauged $\boldsymbol{A}$-based formulation of eddy current problems and, therefore, the preconditioning technique described in [28] can be immediately applied to the complex symmetric linear system that results from (42) and this then ensures a robust solver that is capable of coping with the large contrasts in the $\tilde{\kappa}$ associated with this problem.

For problems with curved geometry the approach described in [37] is employed, which, for a tetrahedron $T \in \mathcal{T}_{h}$, takes the form

$$
\left.\boldsymbol{x}\right|_{T}=\sum_{v=1}^{4} \lambda_{v} \boldsymbol{x}_{v}+\sum_{E \in \mathcal{E}_{h}^{T}} \boldsymbol{c}^{E}+\sum_{F \in \mathcal{F}_{h}^{T}} \boldsymbol{c}^{F},
$$

where the superscript $T$ on $\mathcal{E}_{h}$ and $\mathcal{F}_{h}$ is used to indicate the edges and faces, respectively, associated with a tetrahedron $T \in \mathcal{T}_{h}$. The first term represents the standard affine mapping where $\lambda_{v} \in W_{h, 1}$ are the standard lowest order $H^{1}$ conforming hat functions, the second and third terms represents edge and face corrections, respectively, which are only present on curved boundary faces. The degree of the polynomial correction is $g$ and the coefficients of the edge and face corrections, $\boldsymbol{c}^{E} \in W_{g+1}^{E}$ and $\boldsymbol{c}^{F} \in W_{g+1}^{F}$ respectively, are determined by solving local $L^{2}$ minimisation problems on the edges and faces of those elements lying on the curved boundary.

\section{NUMERICAL EXAMPLES}

\section{A. Polarisation tensor for a spherical object}

For the case where $B_{\alpha}$ is a sphere of radius $\alpha=0.01 \mathrm{~m}$, with fictional material parameters $\sigma_{*}=5.96 \times 10^{7} \mathrm{~S} \mathrm{~m}^{-1}$ and $\mu_{*}=\mu_{0}$ and angular frequency $133.5 \mathrm{rad} \mathrm{s}^{-1}$, so that $\nu=1$, we present results to indicate the agreement between

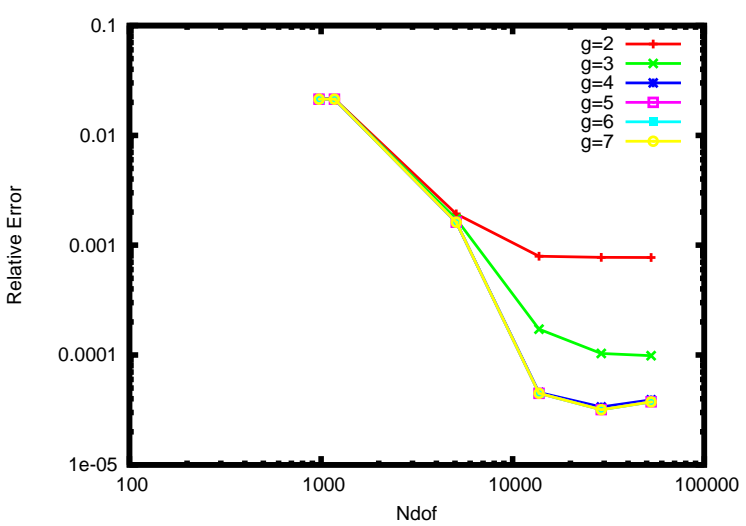

(a)

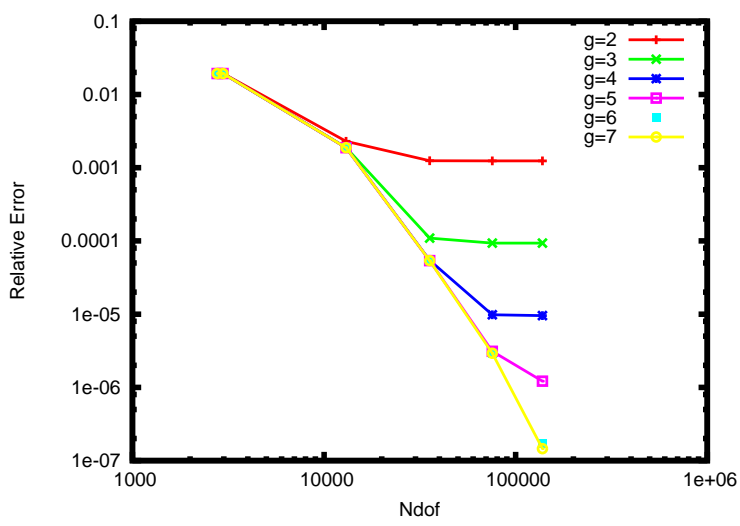

(b)

Fig. 1. Polarisation tensor for a spherical object with $\alpha=0.01 \mathrm{~m}, \sigma_{*}=$ $5.96 \times 10^{7} \mathrm{~S} \mathrm{~m}^{-1}, \mu_{*}=\mu_{0}$ and 133.5rad s${ }^{-1}$ showing convergence of $\left\|\overline{\mathcal{M}}-\overline{\overline{\mathcal{M}}}^{h} p\right\|_{2} /\|\overline{\mathcal{M}}\|_{2}$ with $p=0,1,2,3,4,5$ and $g=2,3,4,5,6,7$ when (a) the domain is truncated at $10 B$ and $(b)$ the domain is truncated at $100 B$.

the numerically calculated polarisation tensor and its known exact solution (which in this case reduces to a scalar multiple of the identity tensor). To compute the numerical tensor, we consider the unit sphere $B$ and choose $\Omega$ to be a sphere which is 10 , and then 100 , times the radius of $B$. For these geometries we generate (coarse) meshes of 880 and 2425 unstructured tetrahedra, respectively, for discretising the two cases. These and subsequent meshes were generated using the NETGEN mesh generator [38]. In order to represent the curved geometry of the sphere polynomial representations using degrees $g=2,3,4,5,6,7$ are considered. We use a regularisation parameter, $\tau$, that is 8 orders of magnitude smaller than $\omega \sigma \mu_{0} \alpha^{2}$. Then, by using elements of order $p=0,1,2,3,4,5$, the approximate solutions $\boldsymbol{\vartheta}_{h p}^{i}, i=1,2,3$, to (42) are computed and, in each case, the error $\left\|\widetilde{\overline{\mathcal{M}}}-\overline{\overline{\mathcal{M}}}{ }^{h}\right\|_{2} /\|\overline{\overline{\mathcal{M}}}\|_{2}$ is evaluated and plot against the number of degrees of freedom (Ndof) in Fig. 1. Note that $\|\cdot\|_{2}$ denotes the entry-wise norm for a rank 2 tensor, $\| \widetilde{\mathcal{M} \|_{2}}=\left(\sum_{\ell m}\left|\overline{\mathcal{M}}_{\ell m}\right|^{2}\right)^{1 / 2}$, and $\overline{\overline{\mathcal{M}}^{h p}}$ is the approximate polarisation tensor computed using $\boldsymbol{\vartheta}_{h p}^{i}$, $i=1,2,3$. In each case, the lines represent the different choices of $g$ and the points on the line represent increasing $p$. In this figure we see that, for truncation at $10 B$, the error associated with the geometry dominates for low $g$, but 


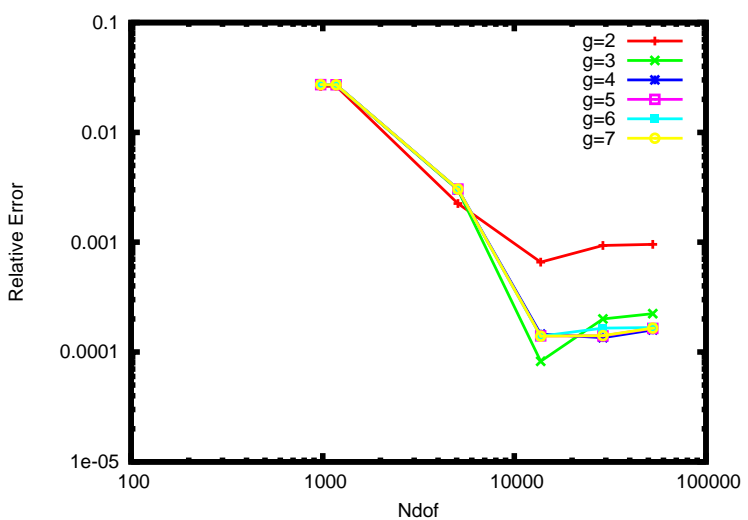

(a)

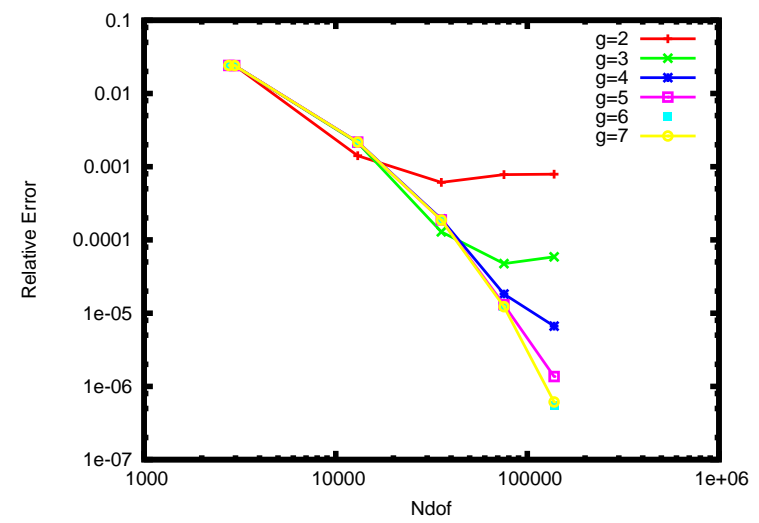

(b)

Fig. 2. Polarisation tensor for a spherical object with $\alpha=0.01 \mathrm{~m}, \sigma_{*}=$ $5.96 \times 10^{7} \mathrm{~S} \mathrm{~m}^{-1}, \mu_{*}=1.5 \mu_{0}$ and $133.5 \mathrm{rad} \mathrm{s}^{-1}$ showing convergence of $\left\|\overline{\mathcal{M}}-\overline{\mathcal{M}}^{h p}\right\|_{2} /\|\overline{\mathcal{M}}\|_{2}$ with $p=0,1,2,3,4,5$ and $g=2,3,4,5,6,7$ when (a) the domain is truncated at $10 \mathrm{~B}$ and $(b)$ the domain is truncated at $100 \mathrm{~B}$.

for $g \geqslant 4$ no further reduction in error can be achieved by increasing $p$ alone. By truncating at $100 B$ and repeating the computations we now see that for $g \geqslant 6$, exponential convergence of the polarisation tensor down to relative errors of $10^{-7}$ results by performing $p$-refinement.

The corresponding results for the same sized object with fictional parameters as before, except $\mu_{*}=1.5 \mu_{0}$, are shown in Fig. 2. For low $g$ and truncation at either $10 B$ or $100 B$, the geometry error dominates but, for sufficiently high $g$ and high $p$, the error can be reduced to less than $10^{-6}$ by increasing the size of the domain to $100 \mathrm{~B}$. In particular, for $g \geqslant 6$ and performing $p$-refinement, exponential convergence of the polarisation tensor is achieved. In general we remark that $\left\|\overline{\mathcal{M}}-\overline{\mathcal{M}^{h} p}\right\|_{2} /\|\widetilde{\mathcal{M}}\|_{2}$ is comprised of the geometry error, which we can reduce by increasing $g$ and/or performing $h-$ refinement, the discretisation error, which is overcome by $p$ and/or $h$-refinement and the outer boundary condition error, which is overcome by increasing the size of the computation domain. Our strategy has the advantage that, in the case of a smooth object, exponential convergence and high levels of accuracy can be achieved by applying $p$-refinement on a coarse mesh, once the geometry has been well resolved and the far field boundary placed sufficiently far from the object.
B. Number of independent coefficients for objects of different shapes and verification of reciprocity

For the objects shown in Table I we have numerically verified that the number of independent coefficients predicted by the theoretical arguments in Section V-A and V-B are correct. We have also numerically verified that (37) holds for a range of objects, dipole positions and moments upto the error term $\Delta$.

\section{Objects in a uniform background field}

For uniform $\boldsymbol{H}_{0}$ we compare $\left(\boldsymbol{H}_{\alpha}-\boldsymbol{H}_{0}\right)(\boldsymbol{x})$ predicted by (24), when $h p$-finite elements are used to numerically compute the rank 2 polarisation tensor $\overline{\mathcal{M}^{h} p} \approx \overline{\overline{\mathcal{M}}}$, with the results obtained by solving the full eddy current problem, using $h p$ finite elements and the formulation in [28]. We undertake this comparison for a series of different shaped objects taken from Table I including when $B_{\alpha}$ is a sphere of radius $0.01 \mathrm{~m}$, a $0.0075 \mathrm{~m} \times 0.015 \mathrm{~m} \times 0.01 \mathrm{~m}$ rectangular block, a cone with height $0.01 \mathrm{~m}$ and maximum radius $0.005 \mathrm{~m}$ and, finally, a cube of side length $0.01 \mathrm{~m}$ with a $0.005 \mathrm{~m} \times 0.005 \mathrm{~m} \times 0.01 \mathrm{~m}$ hole removed.

In each case, we select the far field boundary to be located at distance a 100 times the size of the object, for cases with curved geometries we use $g=4$ and for approximating the solution to (42), we use $p=4$ elements and meshes of 2425 , 3433, 19851 and 7377 unstructured tetrahedra for the cases of a sphere, block, cone and the cube with hole, respectively. We fix the material parameters as $\sigma_{*}=5.96 \times 10^{7} \mathrm{~S} \mathrm{~m}^{-1}$, $\mu_{*}=1.5 \mu_{0}$ and the angular frequency as $133.5 \mathrm{rad} \mathrm{s}^{-1}$ for all objects. The polarisation tensor $\overline{\mathcal{M}^{h} p}$ for each object is computed by considering an appropriate unit-sized object $B$, which, when an appropriate scaling is applied, results in the physical object $B_{\alpha}$. Then, by assuming a uniform incident field $\boldsymbol{H}_{0}(\boldsymbol{x})=\boldsymbol{H}_{0}=\hat{\boldsymbol{e}}_{3}$ we compare $\left|\left(\boldsymbol{H}_{\alpha}-\boldsymbol{H}_{0}\right)(\boldsymbol{x})\right| /\left|\boldsymbol{H}_{0}(\boldsymbol{x})\right|$ when $\boldsymbol{x}=\boldsymbol{r}=r \hat{\boldsymbol{e}}_{i} \in B_{\alpha}^{c}$ and $r \leqslant 0.1 \mathrm{~m}$ and $i=1,2,3$ in turn.

The results of this investigation are shown in Fig. 3. For all cases, the perturbed field predicted by the numerically computed polarisation tensor is in excellent agreement with that obtained by solving the full eddy current problem. In the case of the block, cone and the cube with hole there are some small differences between the perturbed field predicted by the polarisation tensor and that from the full eddy current solution close to the object, but these differences disappear for larger $r$. Indeed this is to be expected as the asymptotic expansion (24) is only valid when $\boldsymbol{x}$ away from $\boldsymbol{z}$. Comparing the perturbed field at a distance of $0.1 \mathrm{~m}$ for the different objects we observe that it's magnitude varies indicating the possibility of being able to determine some characteristics of the shape from the perturbed field measurements. We remark that although higher $p$ (and $g$ ) could be used to compute the polarisation tensor (and the solution to the full eddy current problem) more accurately, the results would be indistinguishable on the chosen scale.

We have also verified that the results predicted by (11), when $h p$-finite elements are used to numerically compute the rank 4 polarisation tensor $\mathcal{M}^{h p} \approx \mathcal{M}$ are identical to 


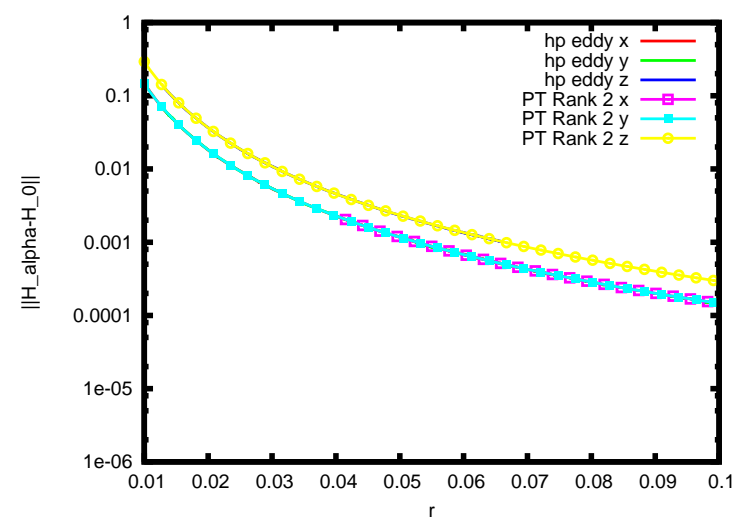

(a)

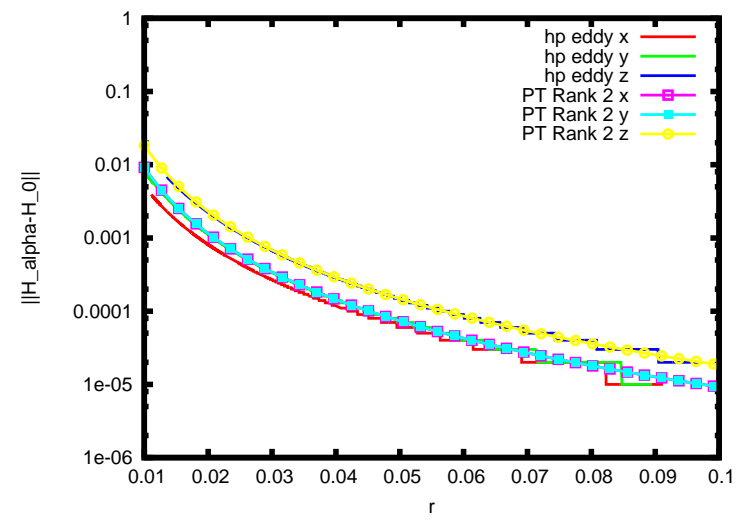

(c)

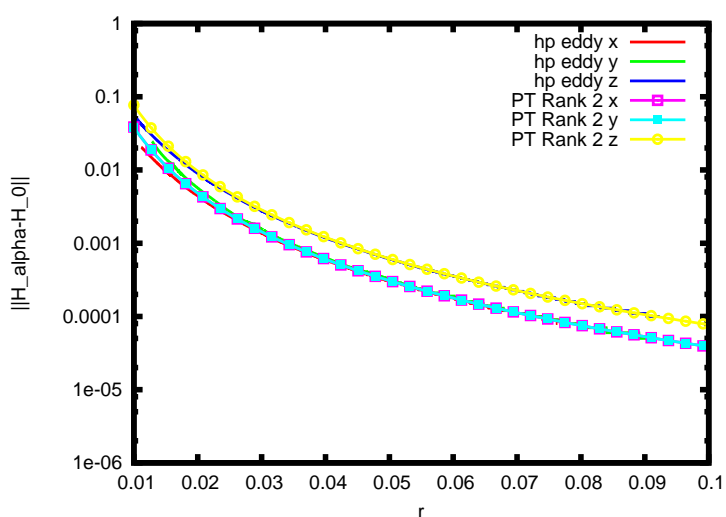

(b)

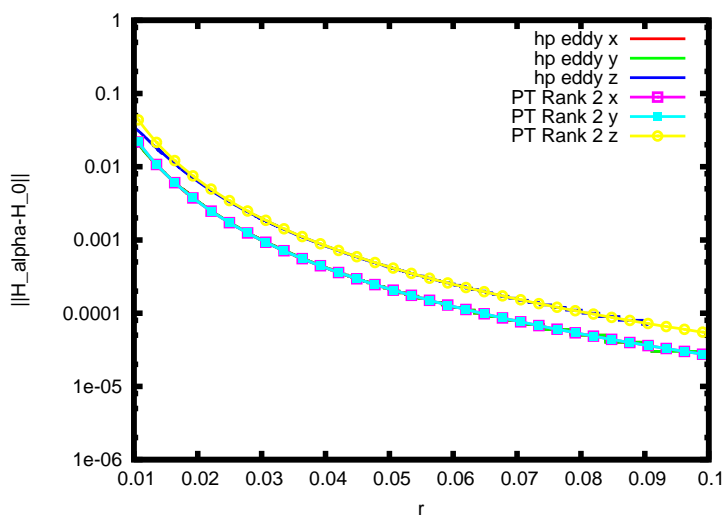

(d)

Fig. 3. Comparison of $\left|\left(\boldsymbol{H}_{\alpha}-\boldsymbol{H}_{0}\right)(\boldsymbol{x})\right| /\left|\boldsymbol{H}_{0}(\boldsymbol{x})\right|$ for uniform $\boldsymbol{H}_{0}(\boldsymbol{x})=\boldsymbol{H}_{0}$ when $\boldsymbol{x}=\boldsymbol{r}=r \hat{\boldsymbol{e}}_{i} \in B_{\alpha}^{c}$ and $r \leqslant 0.1 \mathrm{~m}$ for $i=1,2,3$, in turn, showing the results obtained by using the numerically computed rank 2 polarisation tensor $\overline{\mathcal{M}}^{h}$ and by solving the full eddy current problem when $B_{\alpha}$ is : $(a)$ a sphere of radius $0.01 \mathrm{~m},(b)$ a $0.0075 \mathrm{~m} \times 0.015 \mathrm{~m} \times 0.01 \mathrm{~m}$ rectangular block, $(c)$ a cone with height $0.01 \mathrm{~m}$ and maximum radius $0.005 \mathrm{~m}$ and $(d)$ a cube of side length $0.01 \mathrm{~m}$ with a $0.005 \mathrm{~m} \times 0.005 \mathrm{~m} \times 0.01 \mathrm{~m}$ hole removed.

those predicted by (24), using the computed $\overline{\widetilde{\mathcal{M}^{h}}} \approx \widetilde{\widetilde{\mathcal{M}}}$. The comparisons between $\left(\boldsymbol{H}_{\alpha}-\boldsymbol{H}_{0}\right)(\boldsymbol{x})$ predicted by (24) and solving the full eddy current problem for other objects are similar to those shown.

\section{Objects in a rotational background field}

In this section, we perform a similar comparison to that undertaken in Section IX-C but now with $\boldsymbol{H}_{0}(\boldsymbol{x})$ generated by a coil carrying a current such that $\left|\boldsymbol{J}_{0}\right|=1 \times 10^{6} \mathrm{Am}^{-2}$. The coil is taken to be a torus of inner radius $0.005 \mathrm{~m}$ and outer radius $0.01 \mathrm{~m}$ and has position $0.4 \hat{\boldsymbol{e}}_{3} \mathrm{~m}$ relative to the centre of the object. The shape and material properties of the different objects are as described in Section IX-C. We undertake comparisons of $\left|\left(\boldsymbol{H}_{\alpha}-\boldsymbol{H}_{0}\right)(\boldsymbol{x})\right| /\left|\boldsymbol{H}_{0}(\boldsymbol{x})\right|$ when $\boldsymbol{x}=\boldsymbol{r}=x_{3} \hat{\boldsymbol{e}}_{3} \in B_{\alpha}^{c}$ (L1), $\boldsymbol{x}=\boldsymbol{r}=x_{1} \hat{\boldsymbol{e}}_{1}+x_{3} \hat{\boldsymbol{e}}_{3} \in B_{\alpha}^{c}$ (L2) and $\boldsymbol{x}=\boldsymbol{r}=x_{2} \hat{\boldsymbol{e}}_{2}+x_{3} \hat{\boldsymbol{e}}_{3} \in B_{\alpha}^{c}$ (L3) for $|\boldsymbol{x}| \leqslant 1 \mathrm{~m}$ using $p=4$ elements and $g=4$ when the geometry is curved. The meshes for computing the solution to the full eddy current problem consist of $36012,35347,49086$ and 53743 unstructured tetrahedra for the cases of a sphere, block, cone and the cube with hole, respectively, where the coil has also been discretised in each case.

The results of this investigation are shown in Fig. 4. Note that rather than focusing on the behaviour of the perturbed field along coordinate axes close to the object, as was the case in Fig. 3 for the uniform background field, we now include comparisons in other directions and for larger distances from the object in order to understand the perturbation of the field up to and beyond the position of the coil. In particular, the results show how the normalised field changes with distance along a line directly above the object (L1) and along two diagonal lines extending upwards from the object (L2 and L3). For all objects considered, the agreement between the perturbed field predicted by the asymptotic expansion and (24) using the numerically computed $\widetilde{\mathcal{M}^{h} p}$ and those obtained by solving the full eddy current problem is excellent. We have also verified that the results obtained by (11) using the computed $\mathcal{M}^{h p}$ are identical to those predicted by (24).

The small differences between the results predicted by (24) and solving the full eddy current problem for large $r$ are attributed to the artificial truncation boundary used for solving the full eddy current problem, which, for these examples, has been placed at $r=2 \mathrm{~m}$. These differences are not noticeable in Fig. 3 as the results are shown for small $r$, significantly further away from the artificial truncation boundary. 


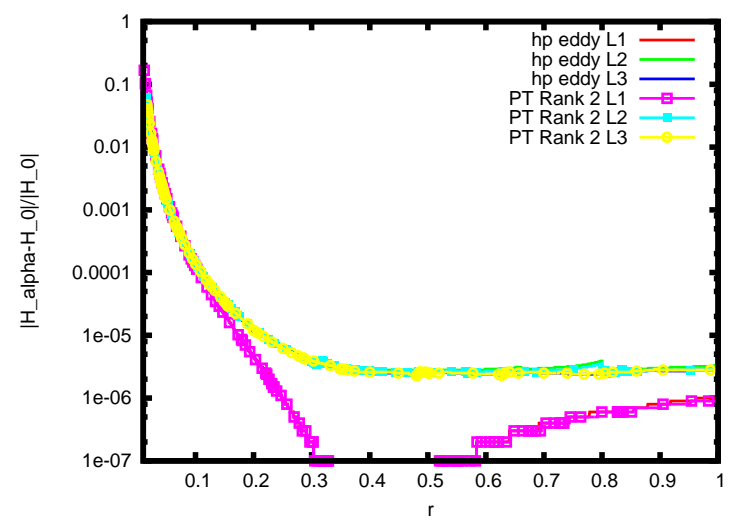

(a)

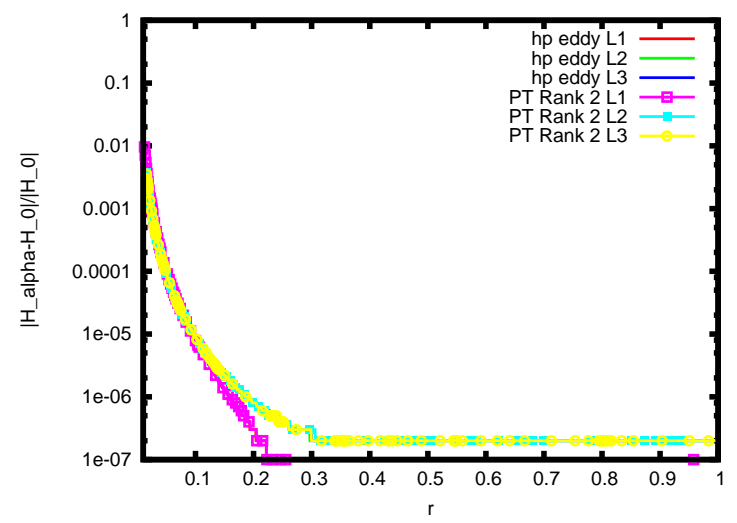

(c)

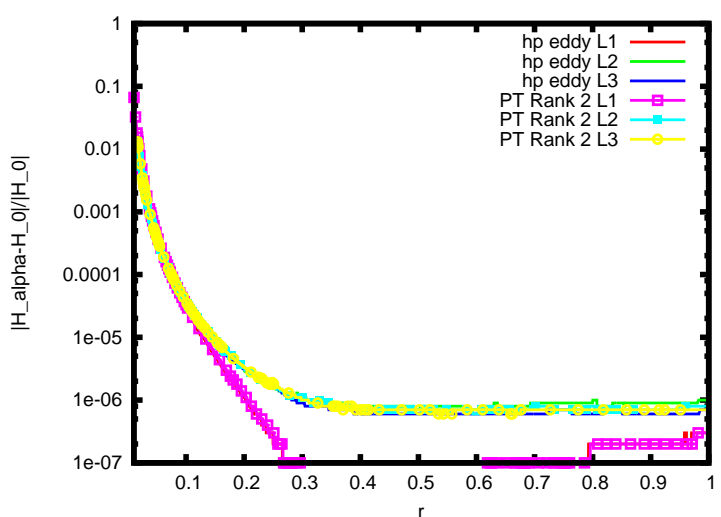

(b)

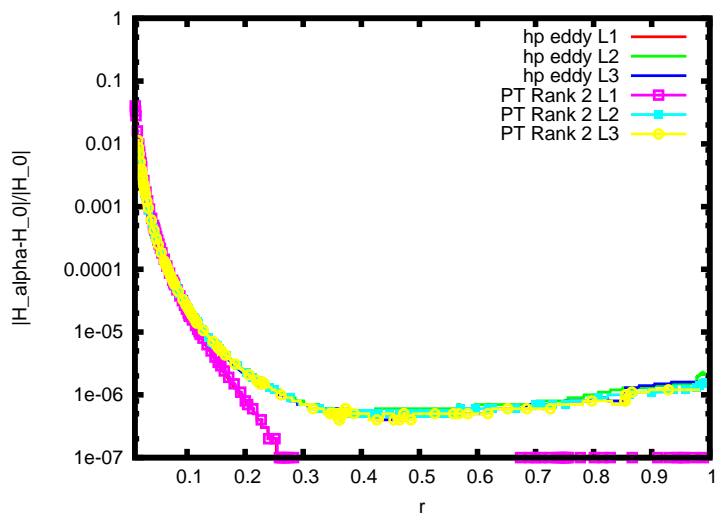

(d)

Fig. 4. Comparison of $\left|\left(\boldsymbol{H}_{\alpha}-\boldsymbol{H}_{0}\right)(\boldsymbol{x})\right| /\left|\boldsymbol{H}_{0}(\boldsymbol{x})\right|$ for rotational $\boldsymbol{H}_{0}$ along the lines L1, L2, and L3, in turn, showing the results obtained by using the numerically computed rank 2 polarisation tensor $\overline{\mathcal{M}}^{h p}$ and by solving the full eddy current problem when $B_{\alpha}$ is : $(a)$ a sphere of radius $0.01 \mathrm{~m},(b)$ a $0.0075 \mathrm{~m} \times 0.015 \mathrm{~m} \times 0.01 \mathrm{~m}$ rectangular block, $(c)$ a cone with height $0.01 \mathrm{~m}$ and maximum radius $0.005 \mathrm{~m}$ and $(d)$ a cube of side length $0.01 \mathrm{~m}$ with a $0.005 \mathrm{~m} \times 0.005 \mathrm{~m} \times 0.01 \mathrm{~m}$ hole removed.

\section{CONCLusion}

In this article we have explained how the recent results of Ammari et al. [26] can be applied to understanding the mystery of the metal detection problem. In doing so we have provided a theoretical footing, which shows that the $\boldsymbol{H}^{M} \cdot\left(\mathcal{M}^{\text {ind }} \boldsymbol{H}^{T}\right)$ type sensitivity that is commonly reported in the literature [9]-[11] is correct, where $\mathcal{M}^{\text {ind }}$ is a rank 2 tensor. To achieve this, we have shown that the rank 4 conductivity tensor, $\mathcal{P}$ obtained by Ammari et al. [26] can be expressed in terms of the rank 3 tensor density $\mathcal{C}$ and this in turn can be expressed in terms of rank 2 tensor $\breve{\mathcal{C}}$. By using properties of these tensors, we have shown that at most 9 independent coefficients are required for defining $\breve{\mathcal{C}}$, and hence $\mathcal{P}$, for a conducting object. A further 9 are required for the rank 2 tensor $\mathcal{N}$ if the object is magnetic. Furthermore, we have shown that the perturbed field for a general object is influenced by a reduced rank 2 symmetric tensor $\overline{\overline{\mathcal{M}}}:=-\breve{\mathcal{C}}+\mathcal{N}$ with just 6 complex independent coefficients, so that $\mathcal{M}^{\text {ind }}=\overline{\overline{\mathcal{M}}}$. If the object has rotational or mirror symmetries we have shown that the number of independent coefficients can be reduced further.

We have included results to illustrate how the tensors can be computed accurately by using the $h p$-finite element method. These results indicate that, for smooth objects, exponential convergence of the computed tensor can be achieved by performing $p$-refinement on a coarse grid with accurate geometry and a far field boundary placed sufficiently far from the object. We have also used these the $h p$-finite element approach to numerically verify the perturbed fields predicted by the asymptotic formula for a range of objects and illuminations and all show excellent agreement when compared with solving the full eddy current problem.

\section{APPENDIX A}

SKEW SYMMETRY OF $\mathcal{C}$

Lemma A.1. The rank 3 tensor density $\mathcal{C}_{m s i}$ is skew symmetric with respect to the first indices and can be represented by just 9 independent coefficients.

Proof. Starting from

$$
\boldsymbol{e}_{i} \times \boldsymbol{\xi}=-\boldsymbol{\theta}_{i}+\frac{\mu_{0}}{\mathrm{i} \nu} \nabla \times \mu_{*}^{-1} \nabla \times \boldsymbol{\theta}_{i},
$$

where the subscript $\xi$ on $\nabla$ has been dropped for simplicity of notation, it follows by application of the alternating tensor that

$$
\xi_{m}=\frac{1}{2} \varepsilon_{k p m} \hat{\boldsymbol{e}}_{k} \cdot\left(-\boldsymbol{\theta}_{p}+\frac{\mu_{0}}{\mathrm{i} \nu} \nabla \times \mu_{*}^{-1} \nabla \times \boldsymbol{\theta}_{p}\right),
$$


in $B$. It is useful to define $\chi_{m}:=\frac{1}{2} \varepsilon_{k p m} \hat{\boldsymbol{e}}_{k}$. $\left(-\boldsymbol{\theta}_{p}+\frac{1}{\mathrm{i} \nu} \nabla \times \tilde{\mu}_{r}^{-1} \nabla \times \boldsymbol{\theta}_{p}\right)$ where $\tilde{\mu}_{r}:=\mu / \mu_{0}$ such that $\tilde{\mu}_{r}=\mu_{r}=\mu_{*} / \mu_{0}$ in $B$ and $\tilde{\mu}_{r}=1$ in $B^{c}$. Note also that $\nabla \times \nabla \times \boldsymbol{\theta}_{i}=\mathbf{0}$ in $B^{c}$ so that $\chi_{m}=-\frac{1}{2} \varepsilon_{k p m} \hat{\boldsymbol{e}}_{k} \cdot \boldsymbol{\theta}_{p}$ in $B^{c}$. Taking this in to consideration then we can write

$$
\begin{aligned}
\mathcal{C}_{m s i}= & \beta \int_{B} \chi_{m} \nabla \chi_{s} \cdot\left(\boldsymbol{\theta}_{i}+\hat{\boldsymbol{e}}_{i} \times \boldsymbol{\xi}\right) \mathrm{d} \boldsymbol{\xi} \\
= & -\frac{\alpha^{3}}{2}\left(\int_{B} \nabla \times\left(\hat{\boldsymbol{e}}_{s} \xi_{m}\right) \cdot \tilde{\mu}_{r}^{-1} \nabla \times \boldsymbol{\theta}_{i} \mathrm{~d} \boldsymbol{\xi}+\right. \\
& \left.\int_{B} \nabla \cdot\left(\tilde{\mu}_{r}^{-1} \nabla \times \boldsymbol{\theta}_{i} \times \chi_{m} \nabla \chi_{s}\right) \mathrm{d} \boldsymbol{\xi}\right) \\
= & -\frac{\alpha^{3}}{2}\left(\int_{B} \hat{\boldsymbol{e}}_{m} \times \hat{\boldsymbol{e}}_{s} \cdot \tilde{\mu}_{r}^{-1} \nabla \times \boldsymbol{\theta}_{i} \mathrm{~d} \boldsymbol{\xi}-\right. \\
& \left.\left.\int_{\Gamma} \chi_{m} \nabla \chi_{s} \cdot \tilde{\mu}_{r}^{-1}\left(\nabla \times \boldsymbol{\theta}_{i} \times \hat{\boldsymbol{n}}^{-}\right)\right|_{-} \mathrm{d} \boldsymbol{\xi}\right) .
\end{aligned}
$$

By using the transmission conditions in (10), $\left[\chi_{m} \nabla \chi_{s} \times \hat{\boldsymbol{n}}\right]_{\Gamma}=$ $\mathbf{0}$ and the fact that the integrand in the last integral can alternatively be written in terms of a tangential trace and a twisted tangential trace, we obtain

$$
\begin{gathered}
\left.\int_{\Gamma} \chi_{m} \nabla \chi_{s} \cdot\left(\tilde{\mu}_{r}^{-1} \nabla \times \boldsymbol{\theta}_{i} \times \hat{\boldsymbol{n}}^{-}\right)\right|_{-} \mathrm{d} \boldsymbol{\xi}= \\
-\int_{\Gamma} \chi_{m} \nabla \chi_{s} \cdot \nabla \times \boldsymbol{\theta}_{i} \times\left.\hat{\boldsymbol{n}}^{+}\right|_{+} \mathrm{d} \boldsymbol{\xi}+ \\
2\left[\tilde{\mu}_{r}^{-1}\right]_{\Gamma} \int_{\Gamma} \chi_{m} \nabla \chi_{s} \cdot \hat{\boldsymbol{e}}_{i} \times \hat{\boldsymbol{n}}^{-} \mathrm{d} \boldsymbol{\xi} .
\end{gathered}
$$

First consider,

$$
\begin{aligned}
& 2\left[\tilde{\mu}_{r}^{-1}\right]_{\Gamma} \int_{\Gamma} \chi_{m} \nabla \chi_{s} \cdot \hat{\boldsymbol{e}}_{i} \times \hat{\boldsymbol{n}}^{-} \mathrm{d} \boldsymbol{\xi} \\
& \quad=2\left[\tilde{\mu}_{r}^{-1}\right]_{\Gamma} \int_{B} \nabla \cdot\left(\chi_{m} \nabla \chi_{s} \times \hat{\boldsymbol{e}}_{i}\right) \mathrm{d} \boldsymbol{\xi} \\
& \quad=2\left[\tilde{\mu}_{r}^{-1}\right]_{\Gamma} \varepsilon_{k s i} \int_{B} \nabla \xi_{m} \cdot \nabla \xi_{k} \mathrm{~d} \boldsymbol{\xi}=2\left[\tilde{\mu}_{r}^{-1}\right]_{\Gamma}|B| \varepsilon_{m s i}
\end{aligned}
$$

by the properties of $\chi_{m}$ in $B$. Secondly, noting that $\boldsymbol{\theta}_{p}=$ $O\left(|\boldsymbol{\xi}|^{-2}\right)$ and $\nabla \times \boldsymbol{\theta}_{p}=O\left(|\boldsymbol{\xi}|^{-3}\right)$ as $|\boldsymbol{\xi}| \rightarrow \infty$ [26] it follows that $\chi_{m}=O\left(|\boldsymbol{\xi}|^{-2}\right.$ ) and $\nabla \chi_{m}=O\left(|\boldsymbol{\xi}|^{-3}\right.$ ) (since $\boldsymbol{\theta}_{p}$ solves a Laplace equation with appropriate decay conditions in an unbounded domain exterior to a sufficiently large sphere that encloses $B$ in a similar way to Proposition 3.1 in [29]). Then, we can apply integration by parts to

$$
\begin{aligned}
& \int_{B^{c}} \chi_{m} \nabla \chi_{s} \cdot \nabla \times \nabla \times \boldsymbol{\theta}_{i} \mathrm{~d} \boldsymbol{\xi}=0 \\
&=\int_{B^{c}} \nabla \chi_{m} \times \nabla \chi_{s} \cdot \nabla \times \boldsymbol{\theta}_{i} \mathrm{~d} \boldsymbol{\xi}- \\
& \int_{\Gamma}^{\chi_{m}} \nabla \chi_{s} \cdot \nabla \times \boldsymbol{\theta}_{i} \times\left.\hat{\boldsymbol{n}}^{+}\right|_{+} \mathrm{d} \boldsymbol{\xi},
\end{aligned}
$$

where the aforementioned decay conditions imply that the far field integral drops out. By rearrangement and inserting (48) and (47) into (46) we have

$$
\begin{aligned}
& \left.\int_{\Gamma} \chi_{m} \nabla \chi_{s} \cdot\left(\nabla \times \boldsymbol{\theta}_{i} \times \hat{\boldsymbol{n}}^{-}\right)\right|_{-} \mathrm{d} \boldsymbol{\xi}= \\
& -\int_{B^{c}} \nabla \chi_{m} \times \nabla \chi_{s} \cdot \nabla \times \boldsymbol{\theta}_{i} \mathrm{~d} \boldsymbol{\xi}+2\left[\tilde{\mu}_{r}^{-1}\right]_{\Gamma}|B| \varepsilon_{m s i},
\end{aligned}
$$

so that

$$
\begin{aligned}
& \mathcal{C}_{m s i}=-\frac{\alpha^{3}}{2}\left(\int_{B} \hat{\boldsymbol{e}}_{m} \times \hat{\boldsymbol{e}}_{s} \cdot \mu_{r}^{-1} \nabla \times \boldsymbol{\theta}_{i} \mathrm{~d} \boldsymbol{\xi}\right. \\
& \left.+\int_{B^{c}} \nabla \chi_{m} \times \nabla \chi_{s} \cdot \nabla \times \boldsymbol{\theta}_{i} \mathrm{~d} \boldsymbol{\xi}-2\left[\tilde{\mu}_{r}^{-1}\right]_{\Gamma}|B| \varepsilon_{m s i}\right) .
\end{aligned}
$$

Thus $\mathcal{C}_{m s i}=-\mathcal{C}_{s m i}$ as required.

\section{APPENDIX B}

SYMMETRY OF $\widetilde{\mathcal{M}}$

Lemma B.1. The tensor $\breve{\mathcal{C}}$ is complex symmetric if $\mu_{*}=\mu$ and the tensor $\overline{\overline{\mathcal{M}}}=-\check{\mathcal{C}}+\mathcal{N}$ is complex symmetric for a general conducting magnetic object.

Proof. The proof of this result builds on a result stated in a preprint of [26]. We begin by checking that $\breve{\mathcal{C}}$ is complex symmetric, to do so we consider

$$
\int_{B} \boldsymbol{\xi} \times\left(\boldsymbol{\theta}_{i}+\hat{\boldsymbol{e}}_{i} \times \boldsymbol{\xi}\right) \mathrm{d} \boldsymbol{\xi} \cdot \hat{\boldsymbol{e}}_{j}=\int_{B}\left(\boldsymbol{\theta}_{i}+\hat{\boldsymbol{e}}_{i} \times \boldsymbol{\xi}\right) \cdot \hat{\boldsymbol{e}}_{j} \times \boldsymbol{\xi} \mathrm{d} \boldsymbol{\xi}
$$

by properties of the scalar triple product. Thus, by using (10),

$$
\begin{gathered}
\int_{B}\left(\boldsymbol{\theta}_{i}+\hat{\boldsymbol{e}}_{i} \times \boldsymbol{\xi}\right) \cdot \hat{\boldsymbol{e}}_{j} \times \boldsymbol{\xi} \mathrm{d} \boldsymbol{\xi}=\frac{\mu_{0}}{\nu \mu_{*}} \int_{B} \nabla \times \nabla \times \boldsymbol{\theta}_{i} \cdot \hat{\boldsymbol{e}}_{j} \times \boldsymbol{\xi} \mathrm{d} \boldsymbol{\xi} \\
=\frac{1}{\mathrm{i} \nu} \int_{B} \frac{1}{\tilde{\mu}_{r}} \nabla \times \nabla \times \boldsymbol{\theta}_{i} \cdot\left(\frac{1}{\mathrm{i} \nu \tilde{\mu}_{r}} \nabla \times \nabla \times \boldsymbol{\theta}_{j}-\boldsymbol{\theta}_{j}\right) \mathrm{d} \boldsymbol{\xi},
\end{gathered}
$$

where $\tilde{\mu}_{r}$ is as defined in Appendix A. Performing integration by parts

$$
\begin{gathered}
\int_{B} \frac{1}{\tilde{\mu}_{r}} \nabla \times \nabla \times \boldsymbol{\theta}_{i} \cdot \boldsymbol{\theta}_{j} \mathrm{~d} \boldsymbol{\xi}=\int_{B} \frac{1}{\tilde{\mu}_{r}} \nabla \times \boldsymbol{\theta}_{j} \cdot \nabla \times \boldsymbol{\theta}_{i} \mathrm{~d} \boldsymbol{\xi}- \\
\int_{\Gamma} \boldsymbol{\theta}_{j} \cdot \frac{1}{\tilde{\mu}_{r}} \nabla \times \boldsymbol{\theta}_{i} \times\left.\hat{\boldsymbol{n}}^{-}\right|_{-} \mathrm{d} \xi
\end{gathered}
$$

Next, using the transmission conditions on $\Gamma$ in (10), then

$\int_{B}\left(\boldsymbol{\theta}_{i}+\hat{\boldsymbol{e}}_{i} \times \boldsymbol{\xi}\right) \cdot \hat{\boldsymbol{e}}_{j} \times \boldsymbol{\xi} \mathrm{d} \boldsymbol{\xi}=$

$\frac{1}{(\mathrm{i} \nu)^{2}} \int_{B} \frac{1}{\tilde{\mu}_{r}^{2}} \nabla \times \nabla \times \boldsymbol{\theta}_{i} \cdot \nabla \times \nabla \times \boldsymbol{\theta}_{j} \mathrm{~d} \boldsymbol{\xi}$

$-\frac{1}{\mathrm{i} \nu} \int_{B} \frac{1}{\tilde{\mu}_{r}} \nabla \times \boldsymbol{\theta}_{i} \cdot \nabla \times \boldsymbol{\theta}_{j} \mathrm{~d} \boldsymbol{\xi}+\frac{1}{\mathrm{i} \nu} \int_{\Gamma} \boldsymbol{\theta}_{j} \cdot \nabla \times \boldsymbol{\theta}_{i} \times\left.\hat{\boldsymbol{n}}^{-}\right|_{+} \mathrm{d} \boldsymbol{\xi}$

$+\frac{2\left[\tilde{\mu}_{r}^{-1}\right]_{\Gamma}}{\mathrm{i} \nu} \int_{\Gamma} \hat{\boldsymbol{e}}_{i} \times \hat{\boldsymbol{n}}^{-} \cdot \boldsymbol{\theta}_{j} \mathrm{~d} \boldsymbol{\xi}$

Then, by performing integration by parts in $B^{c}$,

$$
\begin{aligned}
& \int_{B^{c}} \boldsymbol{\theta}_{j} \cdot \nabla \times \nabla \times \boldsymbol{\theta}_{i} \mathrm{~d} \boldsymbol{\xi}=0 \\
& =\int_{B^{c}} \nabla \times \boldsymbol{\theta}_{i} \cdot \nabla \times \boldsymbol{\theta}_{j}+\int_{\Gamma} \boldsymbol{\theta}_{j} \cdot \nabla \times \boldsymbol{\theta}_{i} \times\left.\hat{\boldsymbol{n}}^{-}\right|_{+} \mathrm{d} \boldsymbol{\xi},
\end{aligned}
$$

which is valid given the decay conditions on $\boldsymbol{\theta}_{i}$ and $\nabla \times \boldsymbol{\theta}_{i}$ as $|\boldsymbol{\xi}| \rightarrow \infty$. [26]. Using (55) in (54) and recalling (15) and (51), then we have that

$$
\begin{aligned}
& \breve{\mathcal{C}}_{j i}=-\frac{\alpha^{3}}{4 \mathrm{i} \nu} \int_{B} \frac{1}{\tilde{\mu}_{r}^{2}} \nabla \times \nabla \times \boldsymbol{\theta}_{i} \cdot \nabla \times \nabla \times \boldsymbol{\theta}_{j} \mathrm{~d} \boldsymbol{\xi} \\
& +\frac{\alpha^{3}}{4} \int_{B \cup B^{c}} \frac{1}{\tilde{\mu}_{r}} \nabla \times \boldsymbol{\theta}_{i} \cdot \nabla \times \boldsymbol{\theta}_{j} \mathrm{~d} \boldsymbol{\xi} \\
& -\frac{\alpha^{3}\left[\tilde{\mu}_{r}^{-1}\right]_{\Gamma}}{2} \int_{\Gamma} \hat{\boldsymbol{e}}_{i} \times\left.\hat{\boldsymbol{n}}^{-} \cdot \boldsymbol{\theta}_{j}\right|_{-} \mathrm{d} \boldsymbol{\xi},
\end{aligned}
$$


which is symmetric when $\mu_{*}=\mu_{0}$. We rewrite $\mathcal{N}_{j i}$ in the following form

$$
\begin{aligned}
\mathcal{N}_{j i} & =\alpha^{3}\left(1-\frac{\mu_{0}}{\mu_{*}}\right) \int_{B}\left(\hat{\boldsymbol{e}}_{i}+\frac{1}{2} \nabla \times \boldsymbol{\theta}_{i}\right) \mathrm{d} \boldsymbol{\xi} \cdot \hat{\boldsymbol{e}}_{j} \\
& =\alpha^{3}\left[\tilde{\mu}_{r}^{-1}\right]_{\Gamma}\left(\int_{B} \hat{\boldsymbol{e}}_{i} \cdot \hat{\boldsymbol{e}}_{j} \mathrm{~d} \boldsymbol{\xi}+\frac{1}{2} \int_{\Gamma} \hat{\boldsymbol{e}}_{j} \times\left.\hat{\boldsymbol{n}}^{-} \cdot \boldsymbol{\theta}_{i}\right|_{-} \mathrm{d} \boldsymbol{\xi}\right) .
\end{aligned}
$$

It then follows that $\overline{\widetilde{\mathcal{M}}}=-\breve{\mathcal{C}}+\mathcal{N}$ can be written as

$$
\begin{aligned}
& \overline{\overline{\mathcal{M}}}_{j i}=\frac{\alpha^{3}}{4 \mathrm{i} \nu} \int_{B} \frac{1}{\tilde{\mu}_{r}^{2}} \nabla \times \nabla \times \boldsymbol{\theta}_{i} \cdot \nabla \times \nabla \times \boldsymbol{\theta}_{j} \mathrm{~d} \boldsymbol{\xi} \\
& -\frac{\alpha^{3}}{4} \int_{B \cup B^{c}} \frac{1}{\tilde{\mu}_{r}} \nabla \times \boldsymbol{\theta}_{i} \cdot \nabla \times \boldsymbol{\theta}_{j} \mathrm{~d} \boldsymbol{\xi} \\
& +\alpha^{3}\left[\tilde{\mu}_{r}^{-1}\right]_{\Gamma}\left(\frac{1}{2} \int_{\Gamma} \hat{\boldsymbol{e}}_{i} \times\left.\hat{\boldsymbol{n}}^{-} \cdot \boldsymbol{\theta}_{j}\right|_{-} \mathrm{d} \boldsymbol{\xi}+\int_{B} \hat{\boldsymbol{e}}_{i} \cdot \hat{\boldsymbol{e}}_{j} \mathrm{~d} \boldsymbol{\xi}\right. \\
& \left.\frac{1}{2} \int_{\Gamma} \hat{\boldsymbol{e}}_{j} \times\left.\hat{\boldsymbol{n}}^{-} \cdot \boldsymbol{\theta}_{i}\right|_{-} \mathrm{d} \boldsymbol{\xi}\right),
\end{aligned}
$$

which is symmetric.

\section{ACKNOWLEDGMENT}

The authors are very grateful to Professors H. Ammari, J. Chen and D. Volkov for their helpful discussions and comments and would also like to thank EPSRC for the financial support received from the grants EP/K00428X/1 and $\mathrm{EP} / \mathrm{K} 039865 / 1$.

\section{REFERENCES}

[1] H. Griffiths, "Magnetic induction tomography," in Electrical Impedance Tomography - Methods, History and Applications, D. Holder, Ed. IOP Publishing, 2005, pp. 213-238.

[2] M. Zolgharni, P. D. Ledger, D. W. Armitage, D. Holder, and H. Griffiths, "Imaging cerebral harmorrhage with magnetic induction tomography: numerical modelling," Physiological Measurement, vol. 30, pp. 187200, 2009.

[3] M. Soleimani, W. R. B. Lionheart, and A. J. Peyton, "Image reconstruction for high contrast conductivity imaging in mutual induction tomography for industrial applications," IEEE Transactions on Instrumentation and Measurement, vol. 56, pp. 2024 - 2032, 2007.

[4] P. Gaydecki, I. Silva, B. Ferandes, and Z. Z. Yu, "A portable inductive scanning system for imaging steel-reinforcing bars embedded within concrete," Sensors and Actuators, vol. 84, pp. 25-32, 2000.

[5] J. Simmonds and P. Gaydecki, "Defect detection in embedded reinforcing bars and cables using a multi-coil magnetic field sensor," Measurement Science and Technology, vol. 10, pp. 640-648, 1999.

[6] "United nations landmines factsheet [accessed 26th may 2013]." [Online]. Available: http://www.un.org/cyberschoolbus/banmines/facts.asp

[7] E. Somersalo, D. Isaacson, and M. Cheney, "A linearised inverse boundary value problem for Maxwell's equations," Journal of Computational and Applied Mathematics, vol. 42, pp. 123-136, 1992.

[8] H. Ammari, M. S. Vogelius, and D. Volkov, "Asymptotic formulas for the perturbations in the electromagnetic fields due to the presence of inhomogeneities of small diamater ii. the full Maxwell equations," $J$. Math. Pures Appl., vol. 80, pp. 769-814, 2001.

[9] Y. Das, J. E. McFee, J. Toews, and G. C. Stuart, "Analysis of an electromagnetic induction detector for real-time location of buired objects," IEEE Transactions of Geoscience and Remote Sensing, vol. 28, pp. 278-288, 1990.

[10] S. J. Norton and I. J. Won, "Identificiation of buried unexploded ordance from broadband electromagnetic induction data," IEEE Transaction on Geoscience and Remote Sensing, vol. 39, 2001.

[11] C. E. Baum, "Some characteristics of electric and magnetic diopole antennas for radiating transient pulses," Air Force Weapons Laboratory, Tech. Rep. 125, 1971.
[12] L. A. Marsh, C. Ktisis, A. Järvi, D. Armitage, and A. J. Peyton, "Threedimensional object location and inversion of the magnetic polarisability tensor at a single frequency using a walk-through metal detector," Measurement Science and Technology, vol. 24, p. 045102, 2013.

[13] R. F. Harrington, TIme Harmonic Electromagnetic Fields. McGraw Hill, London, 1961.

[14] W. R. Smythe, Static and Dynamic Electricity. McGraw Hill, New York, 1968.

[15] R. J. Wait, "A conducting sphere in a time-varying magnetic field," Geophysics, vol. 16, pp. 666-672, 1951.

[16] _ - "A conducting permeable sphere in the presence of a coil carrying an oscillating current," Canadian Journal of Physics, vol. 31, pp. 670678, 1953.

[17] R. E. Kleinman, "Far field scattering at low frequencies," Applied Scientific Research, vol. 18, pp. 1-8, 1967.

[18] _ - "Dipole moments and near field potentials," Applied Scientific Research, vol. 27, pp. 335-340, 1973.

[19] J. B. Keller, R. E. Kleinman, and T. B. A. Senior, "Dipole moments in Rayleigh scattering," Journal of the Institute of Mathematical Applications, vol. 9, pp. 14-22, 1972.

[20] R. E. Kleinman and T. B. A. Senior, "Rayleigh scattering," University of Michigian Radiation Laboratory, Tech. Rep. RL 728, 1982.

[21] G. Dassios and R. E. Kleinman, Low Frequency Scattering. Oxford Science Publications, 2000

[22] H. Ammari and H. Kang, Polarization and Moment Tensors: With Applications to Inverse Problems. Springer, 2007.

[23] C. E. Baum, "Low-frequency near-field magnetic scattering from highly, but not perfectly, conducting bodies," Phillips Laboratory, Tech. Rep. 499, 1993.

[24] H. Ammari and D. Volkov, "The leading-order term in the aymptotic expansion of the scattering amplitude of a collection of finite number dielectric inhomogeneities of small diameter," International Journal for Multiscale Computational Engineering, vol. 3, pp. 149-160, 2005.

[25] P. D. Ledger and W. R. B. Lionheart, "The perturbation of electromagnetics fields at distances large compared to the size of the object," 2012, submitted.

[26] H. Ammari, J. Chen, Z. Chen, J. Garnier, and D. Volkov, "Target detection and characterization from electromagnetic induction data," Journal de Mathématiques Pures et Appliqués, 2013. [Online]. Available: http://dx.doi.org/10.1016/j.matpur.2013.05.002

[27] H. Ammari, J. Chen, Z. Chen, D. Vollkov, and H. Wang, "Detection and classification from electromagnetic induction data," SIAM Journal on Scientific Computing, 2013, submitted.

[28] P. D. Ledger and S. Zaglmayr, " $h p$ finite element simulation of threedimensional eddy current problems on multiply connected domains," Computer Methods in Applied Mechanics and Engineering, vol. 199, pp. 3386-3401, 2010.

[29] H. Ammari, A. Buffa, and J. C. Nédélec, "A justification of eddy currents model for the Maxwell equations," SIAM Journal of Applied Mathematics, vol. 60, pp. 1805-1823, 2000.

[30] K. Schmidt, O. Sterz, and R. Hiptmair, "Estimating the eddy current error," IEEE Transactions on Magnetics, vol. 44, pp. 686-689, 2008.

[31] J. D. Jackson, Classical Electrodynamics. John Wiley and Sons, 1967.

[32] L. D. Landau, E. M. Lifshitz, and L. P. Pitaevskii, Electrodynamics of Continuous Media. Pergammon Press, 2nd edition, 1984.

[33] F. Bachlinger, U. Langer, and J. Schöberl, "Numerical analysis of nonlinear multiharmonic eddy current problems," Numerische Mathematik, vol. 100, pp. 594-516, 2005.

[34] _ "Efficent solvers for nonlinear time-periodic eddy current problems," Computing and Visualization in Science, vol. 9, pp. 197-207, 2006.

[35] S. Zaglmayr and J. Schöberl, "High order Nédélec elements with local complete sequence properties," COMPEL: The International Jounral for Computation and Mathematics in Electrical and Electronic Engineering, vol. 24, pp. 374-384, 2005.

[36] S. Zaglmayr, "High order finite elements for electromagnetic field computation," Ph.D. dissertation, Institut für Numerische Mathematik, Johannes Kepler Universität, Linz, Austria, 2006.

[37] P. D. Ledger and J. Coyle, "Evidence of exponetial convergence in the computation of Maxwell eigenvalues," Computer Methods in Applied Mechanics and Engineering, pp. 587-604, 2005.

[38] J. Schöberl, "Netgen - an advancing fron $2 \mathrm{~d} / 3 \mathrm{~d}$ mesh generator based on abstract rules," Computing and Visualisation in Science, vol. 1, pp. 41-52, 1997. [Online]. Available: http://sourceforge.net/projects/netgenmesher/ 
Paul Ledger (B.Eng. Civil Engineering with Computational Mechanics, The University of Birmingham, 1998, M.Sc. Computational Modelling and Finite Elements in Engineering Mechanics, Swansea University, 1999 and Ph.D. $h p$ Finite elements for Electromagnetic Scattering, Swansea University, 2002) is an Associate Professor in the College of Engineering, Swansea University. His research interests include high order/hp-finite elements applied to problems in computational electromagnetism, error estimation and adaptivity and the computational solution of coupled and inverse problems.

Bill Lionheart (B.Sc. Applied Maths, 1980, The University of Warwick, Ph.D. Electrical Impedance Tomography, 1990, Oxford Brookes University) is a Professor of Applied Mathematics at The University of Manchester; he has worked on a wide range of inverse problems in medicine and industry including electrical impedance tomography, magnetic induction tomography, $\mathrm{x}$-ray CT and PET. His interest in low frequency electromagnetic imaging lead to his involvement in the civilian land mine clearance charity "Find a Better Way", which he was involved in founding and sits on the Scientific and Users Advisory Panel. 